\title{
Fear of hypoglycaemia in paediatric diabetes: a literature review
}

\author{
ABIRAMY FERNANDO, ${ }^{1}$ VINOD PATEL ${ }^{2}$
}

\begin{abstract}
Background: Type 1 diabetes mellitus is one of the most common chronic childhood illnesses and, despite ongoing technological advances, hypoglycaemia remains an inevitable therapeutic risk. Hypoglycaemia results in unpleasant physiological outcomes, social embarrassment and - in extremis - life-threatening consequences. Overlying this inescapable clinical risk is a fear of this risk, ranging from fleeting to overwhelming, and substantially impacting the trajectory of diabetes.
\end{abstract}

Aim: The aim of this literature review is to identify, summarise and critically appraise works pertaining to the development, impact and management of paediatric fear of hypoglycaemia (FoH).

Methods: A search was conducted on Embase, MEDLINE and PsycINFO for studies published between 2000 and 2020, with cross-referencing searches for articles not detected in the original keyword search. Study quality was assessed using recognised tools, and relevant data were extracted systematically.

Results: Forty-three studies met the inclusion criteria. FoH was a moderate problem throughout the studies, increased by a history of hypoglycaemia and predisposition to psychological stress. There was conflicting evidence on the influence of age, diabetes duration, technology and parental demographics. Some studies showed a significant impact on glycaemic control and quality of life (QoL), more consistently for the latter. Only 13 intervention trials were included, showing mixed success with cutting-edge technology, and decent gains with psychological interventions.

Conclusions: FoH is clearly a ubiquitous issue among some families with type 1 diabetes. Prospective longitudinal stud-

Paediatric Registrar, Warwick Medical School, Warwick University, Warwick, UK

2 Professorial Clinical Teaching Fellow: Diabetes and Clinical Skills; Hon Consultant in Endocrinology and Diabetes, Acute Medicine, Medical Obstetrics, Warwick Medical School, Warwick University, Warwick, UK

Address for correspondence: Dr Abiramy Fernando Paediatric Registrar, Warwick Medical School, University of Warwick, Coventry, CV4 7AL, UK

E-mail: abiramy.fernando@nhs.net

https://doi.org/10.15277/bjd.2021.286 ies are required to assess potential risk factors at diagnosis, monitor for the development of $\mathrm{FoH}$ at regular intervals, and enable a more comprehensive assessment of the long-term impact on glycaemic control and QoL. Further randomised controlled trials must demonstrate the value of technological and psychological therapies in order to make such interventions commonplace offerings for families suffering from intractable fear.

Br J Diabetes 2021;21:36-42

Key words: diabetes, hypoglycaemia, fear, $\mathrm{HbA}_{1 c}$, quality of life

\section{Introduction}

Type 1 diabetes mellitus (T1DM) is one of the most common chronic childhood illnesses, affecting 196 per 100,000 children aged 0-15 years in England and Wales. ${ }^{1}$ Despite rapid technological advances in diabetes therapy, ${ }^{2}$ hypoglycaemia remains the commonest acute complication of diabetes care. ${ }^{3}$ Intensive insulin therapy can increase hypoglycaemic frequency three-fold ${ }^{4}$ and individuals with a $<5$-year T1DM duration experience on average 1.1 severe hypoglycaemic episodes per patient per year. ${ }^{5}$ Hypoglycaemia can result in unpleasant physiological symptoms, social embarrassment and - in extremis - life-threatening consequences. Overlying this inescapable clinical risk is a fear of this risk. This construct has been labelled a fear of hypoglycaemia $(\mathrm{FoH})$ and can substantially impact the trajectory of diabetes. Individuals with strong FoH indulge in compensatory mechanisms to avoid hypoglycaemia, maintaining a 'safe' hyperglycaemia while carrying increased diabetes distress (DD) and poorer quality of life (QoL).

The aim of this literature review is to identify, summarise and critically appraise works pertaining to the development, impact and management of paediatric $\mathrm{FoH}$. This will encompass examining FoH measurement tools, identifying predictive factors, exploring its impact and evaluating minimisation strategies. Prior works include a systematic review in parents of young children (PYC) containing six eligible studies ${ }^{6}$ and a broader review of children, adolescents and parents, comprising 16 studies. ${ }^{7}$ Although both highlighted the significance of FoH, its consequences were not fully explored, technology was less abundant, and paediatric behavioural trials non-existent. The current review aims to clearly delineate the impact of $\mathrm{FoH}$ on glycated haemoglobin $\left(\mathrm{HbA}_{1 \mathrm{c}}\right)$ and $\mathrm{QoL}$, underscoring the need for resource allocation. Moreover, diabetes care has been transformed by a decade of technological innovation, from 
Figure 1. PRISMA flow diagram of study selection process. ${ }^{16}$

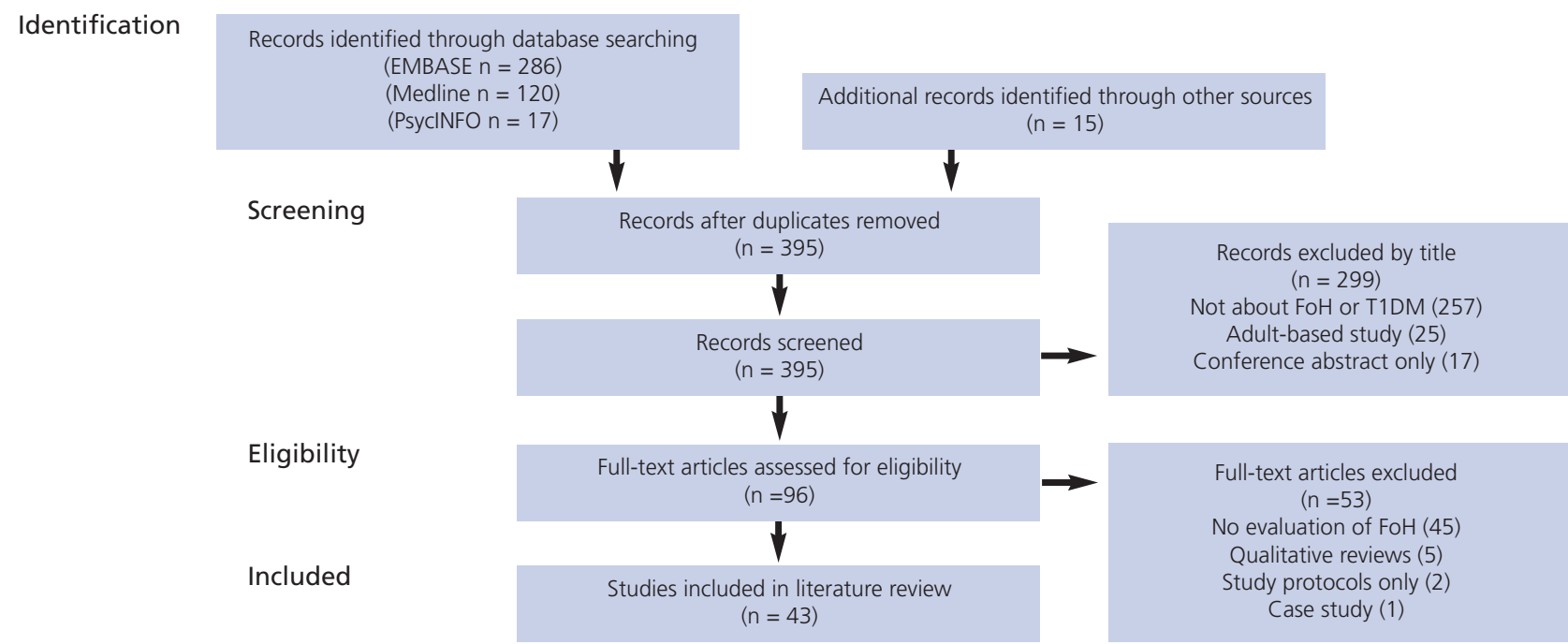

continuous subcutaneous insulin infusions (CSII) and continuous glucose monitors (CGM) to sensor-augmented pump therapy (SAPT) and closed-loop systems, and the debate deserves reinvigorating..$^{8-10}$

\section{Methods}

The research question was generated using the Population, Intervention, Comparator and Outcome (PICO) approach. ${ }^{11}$ The population comprised children and young people (CYP) aged 0-18 years with T1DM or their parents, and whether FoH influenced glycaemic control and QoL. A literature search was conducted on Embase, MEDLINE and PsycINFO. The bibliographies of retrieved papers were also reviewed. Letters to the editor, abstracts and scientific meeting proceedings were excluded. The search was restricted to English language publications from 2000 to 2020, to capture recent changes in technology (see Appendix 1, available online www.bjd-abcd.com, for full search strategy).

Titles and abstracts were examined for inclusion. All study designs meeting PICO parameters were eligible. Exclusion criteria included primarily adult-based studies, a failure to quantitatively assess FoH or either primary outcome. Included studies were critically appraised using recognised tools: the Centre for Evidence Based Medicine criteria for cross-sectional studies, ${ }^{12}$ the National Heart, Lung, Blood Institute checklist for pre-post prospective studies, ${ }^{13}$ and the Critical Appraisal Skills Programme checklist for randomised controlled trials (RCTs) ${ }^{14}$ and systematic reviews ${ }^{15}$ (Appendix 2 available online www.bjd-abcd.com).

Data extracted included study design, demographics, diabetes duration, insulin mode, $\mathrm{HbA}_{1 \mathrm{c}}$, FoH and QoL assessments, hypoglycaemia prevalence, pertinent results, strengths and limitations (Appendix 3 available online www.bjd-abcd.com). Due to differences in populations, treatment regimens and outcome measures, a meta-analysis was not conducted. Instead, a narrative synthesis is presented.

\section{Results}

Search results

Of the 395 abstracts screened, 43 papers were included in the final analysis (see Figure 1).

The majority were cross-sectional studies $(n=28)$, of which two datasets were used twice ${ }^{17-20}$ and three papers aggregated several studies. ${ }^{21-23}$ There were two literature reviews, ${ }^{6,7}$ five pre-post prospective studies and eight RCTs. Sample size ranged from 16 to 549 (mean 142) and 90\% were of Western origin (Figure 2).

Eleven studies investigated parent-child dyads. Nineteen explored parental FoH, with 11 focusing on PYC, facing specific challenges of irregular eating and activities, difficulty matching insulin, greater aberrant glycaemia and subtleties in detection. Seven of 11 studies examining children's FoH explored adolescents, confronting the complexities of puberty, subversion and peer influences.

Figure 2. Geographical distribution of selected studies

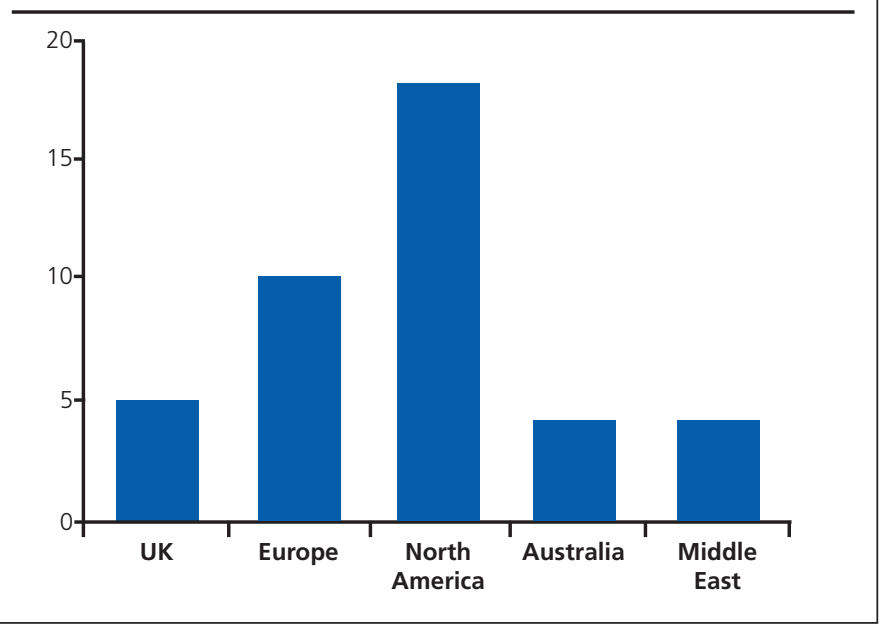




\section{Measurement of fear of hypoglycaemia}

The Hypoglycaemia Fear Survey (HFS) is the most well-established measure assessing FoH, using a worry (HFS-W) and behaviour (HFSB) subscale with 33 items graded from never to always on a Likert scale. ${ }^{24}$ The tool was modified to 25 items for parents (HFS-P) ${ }^{25}$ and revised for PYC (HFS-PYC). ${ }^{26}$ An adaptation for 6-18-year-olds also exists (HFS-C). ${ }^{27}$

The HFS-P demonstrates acceptable reliability with an internal consistency range of 0.88-0.91 for the HFS-W and 0.72-0.76 for the HFS-B. ${ }^{21}$ The HFS-B often displays slightly reduced internal consistency, registering appropriate hypoglycaemia avoidance strategies alongside inappropriate FoH-driven actions. ${ }^{7}$ Modified versions also show sufficient test-retest reliability. ${ }^{21}$ Although less used, the HFS-C has similarly been shown to have an internal consistency of 0.86 and good convergent validity. ${ }^{28} \mathrm{~A}$ key limitation of all HFS versions is the absence of established clinical cut-offs, making clinical interpretability challenging.?

The Children's Hypoglycaemic Index (CHI) is a contemporary alternative, encompassing a fear, situation and behaviour subscale, demonstrating a good internal consistency of 0.89 , decent testretest reliability with a Pearson's correlation coefficient of 0.76 and strong convergent validity among its various subscales. It was purposefully developed for children, explores more areas and comprises FoH-specific behaviours. ${ }^{29}$ However, it is less popular and requires further validation in practice.

\section{Predictors of fear of hypoglycaemia}

Hypoglycaemic frequency and severity is a key factor in FoH development. ${ }^{26,28}$ In a large Australian study of 325 parents of 8-18year-olds, severe hypoglycaemia (SH) conveyed a 6.3 higher HFS-P score $(p=0.004),{ }^{30}$ while a Slovenian work linked SH with maternal hypoglycaemia preventative behaviours $(r=0.25 ; p=0.03) .{ }^{31} \mathrm{SH}$ also positively correlated with HFS-C helplessness scores $(r=0.19$; $\mathrm{p}=0.01$ ) in an aggregated US study of 2596 -18-year-olds. ${ }^{22} \mathrm{SH}$ clearly has a major role in the construct of $\mathrm{FoH}$, although it can of course flourish irrespective of hypoglycaemic experience: in a large US study of PYC, recent SH was wholly unrelated to 549 HFS-P worry scores..$^{32}$ Other studies show adolescent emergency glucose carriage $(F=6.36 ; p<0.05)^{28}$ or diabetes management confidence $(r=0.3 ; p<0.01)$ to be more predictive, ${ }^{33}$ highlighting the ability to deal with $\mathrm{SH}$ to be at least as important as experience of $\mathrm{SH}$ in the development of $\mathrm{FoH}$.

A second hypothesis is that predisposition to stress, anxiety and depression contributes to FoH. ${ }^{34}$ Less mindful parenting was associated with higher HFS-P scores $(p=0.006)$ for 421 Dutch parents, ${ }^{35}$ and a Norwegian study correlated the Hopkins Symptom Checklist-25 (HSCL-25) for depression and anxiety with HFS-P worry scales among 200 mothers $(r=0.04 ; p<0.001)$ and fathers $(r=0.28$; $p=0.006) .{ }^{19}$ Among CYP social anxiety and HFS-C scores positively correlated for North American boys $(r=0.45 ; p<0.01)$ and girls $(r=0.30 ; p<0.005),{ }^{36}$ as did emotional disorders and HFS-B scores among Saudi adolescents. ${ }^{37}$ Of course, such psychological co-morbidities are also associated with certain sociodemographic factors, compounding vulnerability to FoH. For instance, parenting stress has been linked to having younger children, lower socioeconomic status and a non-Caucasian background, factors all also independently associated with FoH. ${ }^{38}$

The most noteworthy demographic variable was gender. Several international studies demonstrated significantly higher maternal HFS scores. ${ }^{18,19,26,31}$ Girls had higher HFS-C helplessness scores $(\mathrm{F}=4.33 ; \mathrm{p}=0.039)$ than boys, ${ }^{22}$ and twice as high $\mathrm{FoH}$ scores $(p<0.0001)$ in a 453-strong adolescent Swedish study. ${ }^{39} \mathrm{Few}$ studies depicted no gender disparity. ${ }^{40}$ Age was also influential: parents of 6-11-year-olds had higher HFS-P scores than parents of children aged $0-5$ years $(p=0.003)$ or $>12$ years $(p=0.003)$, perhaps reflecting care transition from parent to school, ${ }^{40}$ and adolescent age correlated with higher HFS-C social consequence scores. ${ }^{22}$ However, associations between age and FoH were inconsistent. ${ }^{32,41}$ The impact of technology was also indeterminate, ranging from higher HFS-P behaviour scores with multiple daily injections, ${ }^{19}$ and lower HFS-C worry scores with CSII $(p<0.05),{ }^{37}$ to no impact ${ }^{28,42}$ or moderate FoH encouraging CSII use. ${ }^{43}$

\section{Impact of fear of hypoglycaemia}

FoH is postulated to cause hypoglycaemia-avoidant behaviour, prolonged hyperglycaemia, poor glycaemic control and increased $\mathrm{HbA}{ }_{1 \mathrm{c}}$ levels. Hyper-vigilant parents admit to accepting higher target ranges where such vigilance is implausible, ${ }^{44}$ as do adolescents seeking to avoid humiliating public hypoglycaemia. Several studies confirmed significant associations between $\mathrm{FoH}$ scores and HbA 1 c. ${ }^{19,30,31,43}$ Others demonstrated no correlations between HFS-P, $18,26,32$ HFS- $\mathrm{C}^{17,37}$ and $\mathrm{HbA}_{1 c}$. In some cases, despite high maternal HFS-B, ${ }^{45}$ or HFS-C maintain high blood glucose factor scales ${ }^{22}$ correlating with hyperglycaemia, there was no corresponding rise in $\mathrm{HbA}_{1 \mathrm{c}}$. It is clear that $\mathrm{HbA}_{1 \mathrm{c}}$ is a multi-factorial derivation, often poorly reflective of everyday blood glucose excursions. More detailed glycaemic data are required to truly capture the impact of $\mathrm{FoH}$ on glycaemic control. Contrary to the initial hypothesis, $\mathrm{FoH}$ can also intensify diabetes control, negating any negative impact on $\mathrm{HbA}_{1 \mathrm{c}}$ or even improving glycaemic control, ${ }^{21,23}$ although this was a far less common pattern.

The second key FoH impact is upon QoL, although few studies cite QoL as a primary outcome. It is challenging to deduce whether predisposition to stress, anxiety and depression increases $\mathrm{FoH}$, or if FoH intensifies pre-existing psychological burden. In reality, this relationship is bi-directional and there is likely to be an element of reverse causality. ${ }^{34}$ Parents and children in the highest fear quartile have been shown to have lower scores on the Paediatric Quality of Life Inventory (PedsQL) by 20-22\%, 30 and significant associations have been demonstrated between $\mathrm{FoH}$ and DD in adolescent girls $(p=0.044)$ and boys $(p=0.026){ }^{39}$

\section{Minimisation of fear of hypoglycaemia}

The 13 paediatric intervention trials identified highlight the ambiguity of using technology to reduce hypoglycaemia risk and fear. The Juvenile Diabetes Research Foundation CGM RCT failed to exhibit appreciable reductions in HFS-P and HFS-C scores across 10 UK sites ${ }^{46}$ while a smaller UK study of 16 adolescents did show HFS-P (98.69 vs 66.69; $p<0.0021)$ and HFS-C (97.38 vs 59.75; $\mathrm{p}=0.003$ ) reductions with 12 months' $\mathrm{CGM}^{47}$ as did an Australian 
crossover RCT evaluating remote monitoring mobile CGM. ${ }^{48}$ In a multicentre German observational study, CSII use for 6 months conferred significant reductions in HFS-P worry scores ( $d=0.4-0.6$; $\mathrm{p}<0.01){ }^{49}$ with replicable results a decade later, ${ }^{50}$ and in Saudi Arabia, flash glucose monitoring improved adolescent HFS-C scores $(p=0.0001){ }^{51}$ A multicentre crossover RCT involving Israel, Slovenia and Germany comparing an artificial pancreas system with SAPT for 4 nights demonstrated significant HFS-C worry reductions (1.04 vs $0.90 ; p=0.017$ ), ${ }^{52}$ whereas a UK crossover RCT comparing closed loop systems with SAPT did not, ${ }^{53}$ nor did a multicentre Australian RCT comparing predictive low glucose management versus SAPT. ${ }^{54}$

A comprehensive adult literature review showcased blood glucose awareness training and cognitive behavioural therapy (CBT) as effective interventions..$^{55} \mathrm{~A}$ US multisite RCT involving 258 adolescents evaluated the Flexible Lifestyles Empowering Change (FLEX) programme of motivational interviewing and problemsolving skills. Significant improvements were found in adolescent worry/helplessness criteria $(-0.16 ; p=0.04)$, adolescent healthrelated QoL (3.18; $\mathrm{p}=0.009)$ and parents' behaviours to maintain high blood glucose $(-0.21 ; \mathrm{p}=0.005) .{ }^{56}$ Another American intervention using video-based telehealth (REDCHiP) involved 36 parents of 2-6-year-olds. REDCHiP comprised a 10-week programme applying CBT principles to recognise FoH-related thoughts and behaviours, refining coping strategies and practising exposures to challenges. At 3 months there were significant reductions in HFSPYC and DD scores. ${ }^{57}$

\section{Discussion}

\section{Main findings}

$\mathrm{FoH}$ is a pervasive problem, dependent on a range of factors. Negative hypoglycaemic experience is clearly key, with psychological comorbidity serving as both a predictive and confounding factor. Greater female $\mathrm{FoH}$ prevalence undoubtedly reflects a higher female psychological burden with double the $\mathrm{DD}^{39}$ and greater anxiety levels, ${ }^{36,37}$ although paternal $\mathrm{FoH}$ is poorly represented with the only dedicated study displaying low $\mathrm{FoH}$ and state anxiety. ${ }^{58}$ FoH often results in deteriorating glycaemic control, which is sometimes reflected in increased $\mathrm{HbA}_{1}$ levels. The impact of $\mathrm{FoH}$ on QoL is also more nuanced, as innumerable variables contribute to QoL, not least of which is chronic illness itself. ${ }^{59}$

Technology has a definitive role in minimising FoH, which is most beneficial in conjunction with psychological gains. Successful intervention studies reveal significant reductions in PedsQL, ${ }^{49}$ parental health-related QoL, stress and anxiety, alongside FoH reductions. ${ }^{48}$ Psychological intervention is clearly vital, but requires significant buy-in. A UK pilot of problem-solving workshops highlighted significant recruitment issues: although over $90 \%$ of the 89 families approached had high HFS-P scores, only $25 \%$ participated, citing reluctance to miss school, lack of time, interest or travel difficulties. ${ }^{60}$ Lessons must be learnt for future directives and further statistically powered RCTs are needed to confirm the validity of this approach.

\section{Strengths and limitations}

The majority of papers were cross-sectional studies, relatively quick, low-cost undertakings, useful in displaying prevalence, associations and new hypotheses, but unable to establish causality or temporality. Only seven studies performed power calculations to justify sample size; others were likely woefully underpowered. Inter-study variability also rendered some comparisons or aggregations redundant. For instance, a third of studies lacked a definition for $\mathrm{SH}$, definitions varied widely, and most SH was self-reported. Only four intervention trials listed $\mathrm{FoH}$ as a primary outcome, nevertheless $92 \%$ provided significant $p$ values with precise confidence intervals. Sadly, all lacked a cost-benefit analysis (Appendix 2).

Although $\mathrm{FoH}$ measurement was largely comparable and robust, with $93 \%$ of studies using the psychometrically strong HFS, this questionnaire is subject to recall bias, requires literacy, selfassessment and abstract reasoning. Age-specific considerations include the ability of younger children to hypothecate, adolescents to be candid and parental engagement in diabetes care. The impact of FoH was chiefly assessed upon $\mathrm{HbA}_{1 c}$ and QoL. The validity of the former was marred by historic clinic records, different laboratories, self-report ${ }^{35}$ and missing data. ${ }^{45}$ It is also likely that time spent in range is a more useful marker than $\mathrm{HbA}_{1 \mathrm{c}}$. QoL was assessed using an array of established tools, limiting comparability, and was coloured with recall bias.

Selection bias was a fundamental limitation: most recruited opportunistically from diabetes clinics or camps, 22 were restricted to single centres and only a handful accessed national registries. . $^{32,35,39}$ Participants were self-selected by virtue of attending clinic, answering calls or adverts, reflecting a motivated cohort. Further commitment involved questionnaire completion, regular self-monitoring of blood glucose or embracing technology. Response rates across 27 studies ranged from 21\% to 96\% (mean 61\%). Engaged respondents generally revealed better glycaemic control than nonrespondents, ${ }^{30,31,39,57}$ with a mean study participant $\mathrm{HbA}_{1 c}$ of 66.6 $\mathrm{mmol} / \mathrm{mol}$ and CSII use of 5-86\%, often deviating markedly from UK rates of $36.7 \% .{ }^{1}$ Studying populations with better glycaemic control potentially skews the FoH burden and its confounders.

Reviewing only English language publications delivered populations fairly reflective of the UK. Middle Eastern studies relied on questionnaire translation and back-translation, 17,18,37,51 as did many European studies. ${ }^{19,20,31,49,50}$ This may have introduced inaccuracies and cultural inconsistencies. Study cohorts reflected narrow socioeconomic groups: 20 of 23 studies describing ethnicity were $71-$ 97\% Caucasian, 15 had a 69-98\% married population and 22 demonstrated higher parental education, employment or income (Appendix 3). This diminishes the wider applicability of the results while highlighting the time, interest and literacy often decisive in study participation. Future studies need selection processes which overcome these biases. Mothers represented $52-98 \%$ of parent participants (mean 80\%) across 20 studies, excluding exclusively maternal or paternal studies. ${ }^{45,58,59}$ Achieving gender parity is challenging, as mothers are usually the primary caregivers whereas fathers undertake $<20 \%$ of diabetes-related tasks. ${ }^{58}$ It is nevertheless important that future studies are more representative.

Implications for future research and practice

There has been a substantial body of work evaluating the scope of 


\section{Key messages}

- Fear of hypoglycaemia can be a significant issue among children and young people with type 1 diabetes and their parents

- Hypoglycaemic experience and psychological vulnerability are core features in the construct

- The impact on glycaemic control and quality of life is potentially considerable for children and their families

- Prospective longitudinal studies are needed to accurately assess the true causality and implications of fear of hypoglycaemia

- Both technological and psychological therapies can be beneficial in its management; however, there is an urgent need for more robust paediatric intervention trials to validate these therapies

paediatric FoH, but to truly capture the natural history of an often transient phenomenon, large-scale prospective longitudinal studies are required. An assessment of $\mathrm{FoH}$ should include the validated HFS and an objective psychological evaluation. The outcome of glycaemic control should be broadened to include CGM data, acute and secondary complications. QoL should be assessed by both subjective questionnaires and objective psychological appraisal. To limit selection bias, studies must aim to include both parents of all patients within a named diabetes centre, with efforts to minimise language and travel barriers. Further statistically powered RCTs must confirm the validity and applicability of interventions. Awareness of FoH should be raised among local paediatric diabetes multidisciplinary teams, CYP and their parents, with a view to including HFS-P, HFS-PYC and HFS-C surveys within the annual diabetes review so at-risk families can be offered appropriate interventions.

\section{Conclusions}

This review indicates that $\mathrm{FoH}$ is an important issue among CYP with T1DM and their parents. There are several factors involved in the development of FoH. Personal experience of hypoglycaemia and psychological vulnerability are core features in the construct, but the weight of these factors depends on a host of other sociodemographic variables. The true causality and burden of $\mathrm{FoH}$ can be better established in prospective longitudinal studies, assessing these potential risk factors at diagnosis and monitoring for the development of $\mathrm{FoH}$ at regular intervals. Significant $\mathrm{FoH}$ can invariably impact diabetes management and glycaemic control; longitudinal results with CGM data will enable a subtler evaluation of this relationship. Study spans over decades can also assess the psychological burden of FoH more comprehensively than snapshot cross-sectional data. Although such studies are costly and susceptible to high dropout rates, they are necessary to accurately define the long-term impact of $\mathrm{FoH}$. This enables at-risk individuals to be identified more readily, and intervention measures to be better tailored. Despite a recent expansion in paediatric $\mathrm{FoH}$ intervention trials, numbers are

still small. A greater volume of such trials, with larger study numbers, are desperately needed to demonstrate the value of technological and psychological therapies in order to make such interventions commonplace offerings for families suffering from intractable fear.

\section{Conflict of interest None.}

Author contributions AF conducted the literature review and wrote the first draft. VP reviewed the content and suggested amendments which AF incorporated.

\section{Funding None.}

\section{References}

1. National Paediatric Diabetes Audit (2018-19) National Paediatric Diabetes Report. Health Quality Improvement Partnership, Royal College of Paediatrics and Child Health. 2018-19: 7. https://www.rcpch.ac.uk/ sites/default/files/2020-03/final_npda_core_report_2018-2019.pdf

2. Diabetes UK. Type 1 Diabetes Technology: A Consensus Guideline. June 2018. https://www.diabetes.org.uk/resources-s3/2018-06/Diabetes $\% 20$ UK\%20consensus $\% 20$ guideline $\% 2$ foror $\% 20$ Type $\% 201 \% 20$ diabetes\%20technology.pdf

3. Abraham MB, Jones TW, Naranjo D, et al. ISPAD Clinical Practice Consensus Guidelines 2018: Assessment and management of hypoglycaemia in children and adolescents with diabetes. Pediatr Diabetes 2018;27:178-92. https://cdn.ymaws.com/www.ispad.org/resource/ resmgr/consensus_guidelines_2018_/12.assessment_and_management.pdf

4. The Diabetes Control and Complications Trial Research Group. Hypoglycaemia in the Diabetes Control and Complications Trial. Diabetes 1997:46(2):271-86. https://doi.org/10.2337/diab.46.2.271

5. UK Hypoglycaemia Study Group. Risk of hypoglycaemia in types 1 and 2 diabetes: effects of treatment modalities and their duration. Diabetologia 2007;50:1140-7. http://dx. doi.org/10.1007/s00125-0070599-y

6. Barnard K, Thomas S, Royle P, Noyes K, Waugh N. Fear of hypoglycaemia in parents of young children with type 1 diabetes: a systematic review. BMC Paediatr 2010;10:50. http://dx.doi.org/10.1186/14712431-10-50

7. Driscoll KA, Raymond J, Naranjo D, Patton SR. Fear of hypoglycaemia in children and adolescents and their parents with type 1 diabetes. Current Diabetes Reports 2016;16(8):77. http://dx.doi.org/10.1007/s11892016-0762-2

8. National Institute for Clinical Excellence. Continuous subcutaneous insulin infusion for the treatment of diabetes mellitus. Technology appraisal guideline [TA151]. July 2008. https://www.nice.org.uk/guidance/ta151

9. National Institute for Clinical Excellence. Diabetes (type 1 and type 2) in children and young people: diagnosis and management. NICE guideline [NG18]. August 2015. https://www.nice.org.uk/guidance/ng18

10. National Institute for Clinical Excellence. Integrated sensor-augmented pump therapy systems for managing blood glucose levels in type 1 diabetes (the MiniMed Paradigm Veo system and the Vibe and G4 PLATINUM CGM system). Diagnostics guidance [DG21]. February 2016. https://www.nice.org.uk/guidance/dg21/resources/integrated-sensoraugmented-pump-therapy-systems-for-managing-blood-glucose-levels-in-type-1-diabetes-the-minimed-paradigm-veo-system-and-the-vibeand-g4-platinum-cgm-system-pdf-1053685217221

11. Huang X, Lin K, Demner-Fushman D. Evaluation of PICO as a knowledge representation for clinical questions. AMIA Annual Symposium Proceedings Archive 2006;359-63.

12. Center for Evidence Based Management. Critical Appraisal Checklist for Cross-Sectional Study. 2014. https://cebma.org/wp-content/uploads/ Critical-Appraisal-Questions-for-a-Cross-Sectional-Study-July-20141.pdf

13. National Heart, Lung and Blood Institute. Quality Assessment Tool for Before-After (Pre-Post) Studies with No Control Group. 2018. https://www.nhlbi.nih.gov/health-topics/study-quality-assessment-tools

14. Critical Appraisal Skills Programme. CASP Randomised Controlled Trial Checklist. 2018. https://casp-uk.net/wp-content/uploads/2018/03/ 
CASP-Randomised-Controlled-Trial-Checklist-2018_fillable_form.pdf

15. Critical Appraisal Skills Programme. CASP Systematic Review Checklist. 2018. https://casp-uk.net/wp-content/uploads/2018/03/CASP-Systematic-Review-Checklist-2018_fillable-form.pdf

16. Moher D, Liberati A, Tetzlaff J, Altman DG, the PRISMA Group. Preferred reporting items for systematic reviews and meta-analyses: the PRISMA statement. BMJ 2009;339:b2535. http://dx.doi.org/10.1136/bmj.b2535

17. Amiri F, Mohammadreza V, Gonder-Frederick L. Glycemic control, selfefficacy and fear of hypoglycaemia among Iranian children with type 1 diabetes. Can J Diabetes 2015;39(4):302-7. http://dx.doi.org/10.1016/ j.jcjd.2014.12.011

18. Amiri F, Vafa M, Gonder-Frederick L, et al. Evaluating fear of hypoglycaemia, Pediatric parenting stress, and self-efficacy among parents of children with type 1 diabetes and their correlation with glycemic control. Med J Islamic Republic of Iran 2018;32(1):119. http://dx.doi.org/ 10.14196/mjiri.32.119

19. Haugstvedt A, Wentzel-Larsen T, Graue M, Sovik O, Rokne B. Fear of hypoglycaemia in mothers and fathers of children with type I diabetes is associated with poor glycaemic control and parental emotional distress: a population-based study. Diabet Med 2010;27(1):72-8. http://dx.doi.org/10.1111/j.1464-5491.2009.02867.x

20. Haugstvedt A, Wentzel-Larsen, Aarflot M, Rokne B, Graue M. Assessing fear of hypoglycaemia in a population-based study among parents of children with type 1 diabetes: psychometric properties of the hypoglycaemic fear survey- parent version. BMC Endocrine Disord 2015;15(2). http://dx.doi.org/10.1186/1472-6823-15-2

21. Gonder-Frederick L, Nyer M, Shepard JA, Vajda K, Clarke W. Assessing fear of hypoglycaemia in children with type 1 diabetes and their parents. Diabetes Manage 2011;1(6):627-39. http://dx. doi.org/10.2217/dmt.11.60

22. Shepard JA, Vajda K, Nyer M, Larke W, Gonder-Frederick L. Understanding the construct of fear of hypoglycaemia in pediatric type 1 diabetes. $J$ Pediatr Psycho/ 2014;39(10):1115-25. http://dx.doi.org/10.1093/jpepsy/jsu068

23. Patton SR, Noser AE, Clements MA, Dolan LM, Powers SW. Reexamining the hypoglycemia dear survey for parents of young children in a sample of children using insulin pumps. Diabetes Technol Ther 2017;19(2):1038. http://dx.doi.org/10.1089/dia.2016.0389

24. Cox DJ, Irvine A, Gonder-Frederick L, Nowacek G, Butterfield J. Fear of hypoglycaemia: quantification, validation and utilization. Diabetes Care 1987;10(5):617-21. http://dx.doi.org/10.2337/diacare.10.5.617

25. Clarke WL, Gonder-Frederick A, Snyder AL, Cox DJ. Maternal fear of hypoglycaemia in their children with insulin dependent diabetes mellitus. J Pediatr Endocrinol Metab 1998;11:189-94. http://dx.doi.org/10.1515/ jpem.1998.11.s1.189

26. Patton SR, Dolan LM, Henry R, Powers SW. Fear of hypoglycaemia in parents of young children with type 1 diabetes mellitus. J Clin Psychol Med Settings 2008;15(3):252-9. http://dx.doi.org/10.1007/s10880-0089123-x

27. Green LB, Wysocki T, Reineck BM. Fear of hypoglycaemia in children and adolescents with diabetes. J Paediatr Psychol 1990;15(5):633-41. http://dx.doi.org/10/1111./j.1399-5448.2006.00182.x

28. Gonder-Frederick LA, Fisher CD, Ritterband LM, et al. Predictors of fear of hypoglycaemia in adolescents with type 1 diabetes and their parents. Pediatr Diabetes 2006;7(4):215-22. http://dx.doi.org/10.1111/j.13995448.2006.00182.x

29. Kamps L, Roberts MC, Varela RE. Development of a new fear of hypoglycaemia scale: preliminary results. J Pediatr Psychol 2005;30(3):28791. http://dx.doi.org/10.1093/jpepsy/jsi038

30. Johnson SR, Cooper MN, Davis EA, Jones TW. Hypoglycaemia, fear of hypoglycaemia and quality of life in children with type 1 diabetes and their parents. Diabet Med 2013;30(9):1126-31. http://dx.doi.org/ 10.1111/dme. 12247

31. Pate T, Klemencic S, Battelino T, Bratina N. Fear of hypoglycaemia, anxiety, and subjective well-being in parents of children and adolescents with type 1 diabetes. J Health Psychol 2019;24(2):209-18. http://dx.doi.org/10.1177/1359105316650931

32. Van Name MA, Hilliard ME, Boyle CT, et al. Nighttime is the worst time: parental fear of hypoglycaemia in young children with type 1 diabetes (T1D). Paediatric Diabetes 2018;19(1):114-20. http://dx.doi.org/10.1111/ pedi. 12525

33. Herbert L, Monaghan M, Cogen F, Streisand R. The impact of parents' sleep quality and hypoglycemia worry on diabetes self-efficacy. Behavioral Sleep Med 2015;13(4):308-23. http://dx.doi.org/10.1080/ 15402002.2014.898303

34. Patton SR, Dolan LM, Smith LB, Thomas IH, Powers SW. Pediatric parenting stress and its relation to depressive symptoms and fear of hypoglycaemia in parents of young children with type 1 diabetes mellitus. J Clin Psychol Med Settings 2011;18:345-52. http://dx.doi.org/10.1007/ s10880-011-9256-1

35. Aalders J, Hartman E, Nefs G, et al. Educational and psychological aspects of mindfulness and fear of hypoglycaemia in parents of children with type 1 diabetes: results from Diabetes MILES Youth - The Netherlands. Diabet Med 2018;35(5):650-7. http://dx.doi.org/10.1111/ dme.13594

36. Di Battista AM, Hart TA, Greco L, Gloizer J. Type 1 diabetes among adolescents: reduced diabetes self-care caused by social fear and fear of hypoglycaemia. The Diabetes Educator 2009;35(3):465-75. http://dx.doi.org/10.1177/0145721709333492

37. Al Hayek AA, Robert AA, Braham RB, Issa BA, Sabaan FS. Predictive risk factors of hypoglycaemia and anxiety-related emotional disorders among adolescents with type 1 diabetes. Medical Principles and Practice 2015; 24(3):222-30. http://dx.doi.org/10.1159/000375306

38. Streisand R, Swift E, Wickmark T, Chen R, Holmes C. Pediatric parenting stress among parents of children with type 1 diabetes: the role of selfefficacy, responsibility, and fear. J Pediatr Psychol 2015;30(6):513-21. http://dx.doi.org/10.1093/jpepsy/jsi076

39. Forsander G, Bogelung M, Haas J, Samueslsson U. Adolescent life with diabetes: gender matters for level of distress. Experiences from the National TODS study. Pediatr Diabetes 2017;18(7):651-9. http://dx.doi.org/10.1111/pedi.12478

40. Hawkes CP, McDarby V, Cody D. Fear of hypoglycaemia in parents with type 1 diabetes. J Paediatr Child Health 2014;50(8):639-42. http://dx.doi.org/10.1111/jpc.12621

41. Viaene A, Van Daela T, Bleys D, Faust K, Massa GG. Fear of hypoglycaemia, parenting stress, and metabolic control for children with type 1 diabetes and their parents. J Clin Psychol Med Settings 2017;24:74-81. http://dx.doi.org/10.1007/s10880-017-9489-8

42. Markowitz J, Pratt K, Aggarwal J, Volkening LK, Laffel LMB. Psychosocial correlates of continuous glucose monitoring use in youth and adults with type 1 diabetes and parents of youth. Diabetes Technol Ther 2012; 14(6):523-6. http://dx.doi.org/10.1089/dia.2011.0201

43. Patton SR, Dolan LM, Henry R, Powers SW. Parental fear of hypoglycaemia: young children treated with continuous subcutaneous insulin infusion. Pediatr Diabetes 2007;8(6):362-8. http://dx.doi.org/10.1111/ j.1399-5448.2007.00242.x

44. Lawton J, Waugh N, Barnard KD, et al. Challenges of optimizing glycaemic control in children with type 1 diabetes: a qualitative study of parents' experiences and views. Diabet Med 205;32(8):1063-70. http://dx.doi.org/10.1111/dme.12660

45. Freckleton E, Sharpe L, Mullan B. The relationship between maternal fear of hypoglycaemia and adherence in children with type-1 diabetes. Int $J$ Behav Med 2013;21(5):804-10. http://dx.doi.org/10.1007/s12529-0139360-8

46. Juvenile Diabetes Research Foundation Continuous Glucose Monitoring Study Group. Quality-of-life measures in children and adults with type 1 diabetes. Juvenile Diabetes Research Foundation Continuous Glucose Monitoring randomized trial. Diabetes Care 201;33(10):2175-7. http://dx.doi.org/10.2337/dc10-0331

47. Ng SM, Moore HS, Clemente MF, Pintus D, Soni A. Continuous glucose monitoring in children with type 1 diabetes improves well-being, alleviates worry and fear of hypoglycaemia. Diabetes Technol Ther 2019; 21(3):1-5. http://dx.doi.org/10.1089/dia.2018.0347

48. Burckhardt M, Roberts A, Smith GJ, Abraham MB, Davis EA, Jones TW. The use of continuous glucose monitoring with remote monitoring improves psychosocial measures in parents of children with type 1 diabetes: a randomized crossover trial. Diabetes Care 2018;41(12):2641-3. http://dx.doi.org/10.2337/dc18-0938

49. Muller-Godeffroy E, Treichel S, Wagner VM, German Working Group for Paediatric Pump Therapy. Investigation of quality of life and family burden issues during insulin pump therapy in children with type 1 diabetes mellitus: a large-scale multicentre pilot study. Diabet Med 2009; 
26(5):493-501. http://dx.doi.org/10.1111/j.1464-5491.2009.02707.x

50. Mueller-Godeffroy, Vonthein R, Ludwig-Seibold C, et al, German Working Group for Pediatric Pump Therapy. Psychosocial benefits of insulin pump therapy in children with diabetes type 1 and their families: the Pumpkin multicentre randomized controlled trial. Pediatr Diabetes 2018;19(8):1471-80. http://dx.doi.org/10.1111/pedi.12777

51. Al Hayek AA, Robert AA, Dawish MAA. Evaluation of FreeStyle Libre flash glucose monitoring system on glycemic control, health-related quality of life, and fear of hypoglycaemia in patients with type 1 diabetes. Endocrinol Diabetes 2017;10:1-6. http://dx.doi.org/10.1177/1179551417746957

52. Ziegler C, Lieberman A, Nimri R, et al. Reduced worries of hypoglycaemia, high satisfaction, and increased perceived ease of use after experiencing four nights of MD-Logic artificial pancreas at home (DREAM4). J Diabetes Res 2015;2015:590308. http://dx.doi.org/ 10.1155/2015/590308

53. Barnard KD, Wysocki T, Allen JM, et al. Closing the loop overnight at home setting: psychosocial impact for adolescents with type 1 diabetes and their parents. BMJ Open Diabetes Res Care 2014;2(1):e000025. http://dx.doi.org/10.1136/bmjdrc-2014-000025

54. Abraham MB, Nicholas JA, Smith GJ, et al, PLGM Study Group. Reduction in hypoglycaemia with the predictive low-glucose management system: a long-term randomized controlled trial in adolescents with type 1 diabetes. Diabetes Care 2018;41(2):303-10. http://dx.doi.org/10.2337/ dc17-1604
55. Wild D, Maltzahn R, Brohan E, Christensen T, Clauson P, Gonder-Frederick $L$. A critical review of the literature on fear of hypoglycaemia in diabetes: Implications for diabetes management and patient education. Patient Education and Counselling 2007;68(1):10-15. http://dx.doi.org/ 10.1016/j.pec.2007.05.003

56. Mayer-Davis EJ, Maahs DM, Seid M, et al. Efficacy of the Flexible Lifestyles Empowering Change intervention on metabolic and psychosocial outcomes in adolescents with type 1 diabetes (FLEX): a randomised controlled trial. Lancet Child Adolesc Health 2018;2(9):635-46. http://dx.doi.org/10.1016/S2352-4642(18)30208-6

57. Patton SR, Clements MA, Marker AM, Nelson E. Intervention to reduce hypoglycaemia fear in parents of young kids using video-based telehealth (REDCHiP). Pediatr Diabetes 2019;21(1):112-9. http://dx.doi.org/ 10.1111/pedi.12934

58. Mitchell SJ, Hilliard ME, Mednick L, Henderson C, Cogen FR, Streisand R. Stress among fathers of young children with type 1 diabetes. Family System Health 2009;27(4):314-24. http://dx. doi.org/10.1037/a0018191

59. Grey M. Coping and psychosocial adjustment in mothers of young children with type 1 diabetes. Child Health Care 2009;38(2):91-106. http://dx.doi.org/10.1080/02739610902813229

60. Cai RA, Holt RIG, Casdagli L, et al. Development of an acceptable and feasible self-management group for children, young people and families living with type 1 diabetes. Diabet Med 2017;34(6):813-20. http://dx.doi.org/10.1111/dme.13341

\title{
$\widehat{A B C D} \begin{gathered}\text { Testosterone and Type } 2 \text { Diabetes } \\ \text { Worldwide Audit }\end{gathered}$
}

Association of British Clinical Diabetologists

$A B C D$ has launched a Worldwide Audit of Testosterone and Diabetes in the UK and Internationally to assess real clinical efficacy and safety \& inform future practice and guidelines

Symptomatic Testosterone Deficiency is present in approximately $40 \%$ of men with Type 2 diabetes. Data from patients who are testosterone deficient and not treated can also be entered.

\section{Does your centre diagnose Testosterone Deficiency?}

\author{
If yes, REGISTER YOUR CENTRE!
}

at https://abcd.care/application-join-abcd-worldwide-testosterone-and-diabetes-audit

- you are invited to enter your patients' data into the bespoke online tool

- you will be able to analyse your local data easily

- the data will be automatically added to the national data in anonymised form

- we can provide easy-to-complete paper proformas for use in clinic if preferred

Please remember:

- the more data, the more complete our understanding of Testosterone in real clinical practice

- all contributors will be listed in publications arising from data submission 


\section{Appendix 1. Search Strategies}

\section{A. Embase}

1 type 1 diabetes mellitus.mp. or insulin dependent diabetes mellitus/ (117090)

2 (T1DM or T1D or IDDM).mp. [mp=title, abstract, heading word, drug trade name, original title, device manufacturer, drug manufacturer, device trade name, keyword, floating subheading word, candidate term word] (30663)

31 or $2(122864)$

4 hypoglycemia/ or fear of hypoglycaemia.mp. or fear/ (150043)

5 hypoglycemia/ or FoH.mp. or fear/ (150230)

64 or $5(150251)$

7 HbA1c.mp. or hemoglobin A1c/ (113493)

8 glycosylated hemoglobin/ or glycemic control.mp. or glycemic control/ or glucose blood level/ (323176)

97 or $8(376656)$

10 quality of life.mp. or "quality of life"/ (566645)

11 (depression or anxiety).mp. [mp=title, abstract, heading word, drug trade name, original title, device manufacturer, drug manufacturer, device trade name, keyword, floating subheading word, candidate term word] (879500)

1210 or $11(1366021)$

$13 \quad 3$ and 6 and 9 and 12 (1501)

14 limit 13 to (english language and yr=" 2000 - 2020" and child <unspecified age>) (286)

\section{B. Medline}

type 1 diabetes mellitus.mp or Diabetes Mellitus, Type 1/ (77260)

(T1DM or T1D or IDDM).mp (16468).

1 or $2(80748)$

Hypoglycaemia/ or fear of hypoglycaemia.mp (27064)

Fear/ or FoH.mp. or Hypoglycemia/ (57847)

4 or 5 (57902)

HbA1c.mp. or Glycated Hemoglobin A/ (51919)

Glycated Hemoglobin A/ or Blood Glucose/ or glycemic control.mp. (190137)

7 or 8 (202638)

10 "quality of life.mp. or "Quality of Life"/ (317349)

11 Depression/ or Anxiety/ (525114)

1210 or 11 (803362)

133 and 6 and 9 and 12 (303)

14 Limit 13 to (English language and yr="2000-2020" and "all child (0 to 18 years)" and last 20 years) (120)

\section{Psycinfo}

1 (type 1 diabetes mellitus or diabetes mellitus).mp. [mp=title, abstract, heading word, table of contents, key concepts, original title, tests \& measures, mesh] (11675)

2 (T1DM or T1D or IDDM).mp. [mp=title, abstract, heading word, table of contents, key concepts, original title, tests \& measures, mesh] (785)

31 or $2(11928)$

4 exp Hypoglycemia/ or exp Fear/ or hypoglycaemia.mp. (21131)

5 exp Fear/ or exp Hypoglycemia/ or FoH.mp. (20993)

64 or $5(21147)$

7 HbA1c.mp. (1750)

8 exp Glucose/ or exp Blood Sugar/ or glycemic control.mp. (5846)

97 or $8(7008)$

10 quality of life.mp. or exp "Quality of Life"/ (86860)

11 exp "Quality of Life"/ or QoL.mp. (44348)

12 (depression or anxiety).mp. [mp=title, abstract, heading word, table of contents, key concepts, original title, tests \& measures, mesh] (456241)

1310 or 11 or $12(519192)$

149 or $13(524970)$

153 and 6 and 14 (89)

16 limit 15 to (english language and (childhood <birth to 12 years $>$ or adolescence $<13$ to 17 years $>$ ) and last 20 years) (17) 
Appendix 2. Quality Assessment Tables

A. Cross-Sectional Studies $(n=28)$ CEBM Critical Appraisal of a Cross-Sectional Study (CEBM, 2014)

1. Did the study address a clearly focused question/ issue?

2. Is the research method (study design) appropriate for answering the research question?

3. Is the method of selection of the subjects (employees, teams, divisions, organizations) clearly described?

4. Could the way the sample was obtained introduce (selection) bias?

5. Was the sample of subjects representative with regard to the population to which the findings will be referred?

6. Was the sample size based on pre-study considerations of statistical power?

7. Was a satisfactory response rate achieved?

8. Are the measurements (questionnaires) likely to be valid and reliable?

9. Was the statistical significance assessed?

10. Are confidence intervals given for the main results?

11. Could there be confounding factors that haven't been accounted for?

12. Can the results be applied to your organization? 
Appendix 2. Quality Assessment Tables (continued)

\begin{tabular}{|c|c|c|c|c|c|c|}
\hline Study & 1 & 2 & 3 & 4 & 5 & 6 \\
\hline $\begin{array}{l}\text { First Author } \\
\text { Publication Year }\end{array}$ & $\begin{array}{l}\text { Clearly focused } \\
\text { question }\end{array}$ & $\begin{array}{l}\text { Appropriate } \\
\text { research method }\end{array}$ & $\begin{array}{l}\text { Clear } \\
\text { study design }\end{array}$ & Selection bias & Representative sample & $\begin{array}{l}\text { Power } \\
\text { calculation }\end{array}$ \\
\hline $\begin{array}{l}\text { 1. Aalders } \\
\text { (2018) }\end{array}$ & $\begin{array}{l}\text { Yes } \\
\text { Mindfulness and parental FoH }\end{array}$ & Yes & Yes & $\begin{array}{l}\text { Perhaps } \\
\text { Time commitment } \\
\text { No data on non-participants }\end{array}$ & $\begin{array}{l}\text { Partially of local population } \\
3 \% \text { non-Dutch; } 89 \% \text { married } \\
83 \% \text { paid job }\end{array}$ & No \\
\hline $\begin{array}{l}\text { 2. Al Hayek } \\
\text { (2015) }\end{array}$ & $\begin{array}{l}\text { Yes } \\
\text { FoH and anxiety }\end{array}$ & Yes & Yes & $\begin{array}{l}\text { Yes } \\
\text { Selected from military medical } \\
\text { centre }\end{array}$ & $\begin{array}{l}\text { Reflective of local population } \\
100 \% \text { Arabic }\end{array}$ & No \\
\hline $\begin{array}{l}\text { 3. Amiri } \\
\text { (2014) }\end{array}$ & $\begin{array}{l}\text { Yes } \\
\text { FoH, self-efficacy and } \mathrm{HbA} 1 \mathrm{c}\end{array}$ & Yes & Yes & $\begin{array}{l}\text { Yes } \\
\text { Selected from a limited database }\end{array}$ & $\begin{array}{l}\text { Reflective of local population } \\
100 \% \text { Iranian }\end{array}$ & No \\
\hline $\begin{array}{l}\text { 4. Amiri } \\
\text { (2018) }\end{array}$ & $\begin{array}{l}\text { Yes } \\
\text { FoH, self-efficacy and } \\
\text { parenting stress }\end{array}$ & Yes & Yes & $\begin{array}{l}\text { Yes } \\
\text { Selected from a limited database }\end{array}$ & $\begin{array}{l}\text { Reflective of local population } \\
100 \% \text { Iranian }\end{array}$ & No \\
\hline $\begin{array}{l}\text { 5. Di Battista } \\
\text { (2009) }\end{array}$ & $\begin{array}{l}\text { Yes } \\
\text { Effect of FoH on social } \\
\text { anxiety, adherence and QoL }\end{array}$ & Yes & Yes & $\begin{array}{l}\text { Perhaps } \\
\text { Time commitment } \\
\$ 10 \text { incentive }\end{array}$ & $\begin{array}{l}\text { Representative of clinic population } \\
82 \% \text { white; } 12 \% \text { African-American } \\
\text { but higher SES }\end{array}$ & No \\
\hline $\begin{array}{l}\text { 6. Forsander } \\
\text { (2016) }\end{array}$ & $\begin{array}{l}\text { Yes } \\
\text { Gender and DD, including FoH }\end{array}$ & Yes & Yes & $\begin{array}{l}\text { Perhaps } \\
\text { Time commitment }\end{array}$ & $\begin{array}{l}\text { Partially- national database } \\
69 \% \text { married; } 90 \% \text { economic } \\
\text { status above average, more girls }\end{array}$ & No \\
\hline $\begin{array}{l}\text { 7. Freckleton } \\
\text { (2014) }\end{array}$ & $\begin{array}{l}\text { Yes } \\
\text { Maternal FoH and adherence }\end{array}$ & Yes & Yes & $\begin{array}{l}\text { Yes } \\
\text { Time commitment } \\
\text { Opportunistic from camp/ advert }\end{array}$ & $\begin{array}{l}\text { Yes, most likely } \\
83 \% \text { Australian-born } \\
\text { Little other demographic data }\end{array}$ & No \\
\hline $\begin{array}{l}\text { 8. Frederick } \\
\text { (2011) }\end{array}$ & $\begin{array}{l}\text { Yes } \\
\text { FoH and diabetes self- } \\
\text { management }\end{array}$ & Yes & Yes & $\begin{array}{l}\text { Yes } \\
\text { Time commitment } \\
\text { Opportunistic from clinic/ camp }\end{array}$ & $\begin{array}{l}\text { Yes- diverse sample } \\
66 \% \text { white, } 32 \% \text { black, } 2 \% \\
\text { Hispanic; no SES data }\end{array}$ & No \\
\hline $\begin{array}{l}\text { 9. Gonder-Frederick } \\
\text { (2006) }\end{array}$ & $\begin{array}{l}\text { Yes } \\
\text { Influence of trait anxiety and } \\
\text { hypoglycaemic history on FoH }\end{array}$ & Yes & Yes & $\begin{array}{l}\text { Perhaps } \\
\text { Time commitment with no } \\
\text { compensation }\end{array}$ & $\begin{array}{l}\text { Representative of clinic population } \\
87 \% \text { Caucasian; } 13 \% \text { African-Am } \\
70 \% \text { married; } 75 \%>\text { high school }\end{array}$ & No \\
\hline $\begin{array}{l}\text { 10.Gonder-Frederick } \\
\text { (2011) }\end{array}$ & $\begin{array}{l}\text { Yes } \\
\text { FoH and diabetes control }\end{array}$ & Yes & Yes & Unclear & $88 \%$ Caucasian & No \\
\hline $\begin{array}{l}\text { 11.Grey } \\
\text { (2009) }\end{array}$ & $\begin{array}{l}\text { Yes } \\
\text { Anxiety \& depression in } \\
\text { mothers related to FoH, } \\
\text { coping and metabolic control }\end{array}$ & Yes & Yes & $\begin{array}{l}\text { Yes } \\
\text { Baseline data from an RCT on } \\
\text { coping skills training }\end{array}$ & $\begin{array}{l}\text { Representative of clinic population } \\
85 \% \text { white; } 14 \% \text { Black } \\
55 \% \text { income }>\$ 80,000\end{array}$ & No \\
\hline $\begin{array}{l}\text { 12. Haugstvedt } \\
\text { (2010) }\end{array}$ & $\begin{array}{l}\text { Yes } \\
\text { FoH, hypoglycaemia and } \\
\text { parental emotional distress }\end{array}$ & Yes & Yes & $\begin{array}{l}\text { Perhaps } \\
\text { Time commitment }\end{array}$ & $\begin{array}{l}\text { Representative of clinic population } \\
97 \% \text { Norwegian }\end{array}$ & No \\
\hline $\begin{array}{l}\text { 13. Haugstvedt } \\
(2015)\end{array}$ & $\begin{array}{l}\text { Yes } \\
\text { Examine psychometric } \\
\text { properties of HFS-P }\end{array}$ & Yes & Yes & $\begin{array}{l}\text { Perhaps } \\
\text { Time commitment }\end{array}$ & $\begin{array}{l}\text { Representative of clinic population } \\
87 \% \text { married; } 92 \% \text { men employed } \\
\text { full-time }\end{array}$ & No \\
\hline $\begin{array}{l}\text { 14. Hawkes } \\
(2014)\end{array}$ & $\begin{array}{l}\text { Yes } \\
\text { Parental FoH and glycaemic } \\
\text { control }\end{array}$ & Yes & Yes & $\begin{array}{l}\text { Perhaps } \\
\text { Time commitment }\end{array}$ & $\begin{array}{l}\text { Unclear } \\
\text { Demographics not recorded }\end{array}$ & No \\
\hline $\begin{array}{l}\text { 15. Herbert } \\
(2014)\end{array}$ & $\begin{array}{l}\text { Yes } \\
\text { Relationship of sleep, FoH and } \\
\text { diabetes self-efficacy }\end{array}$ & Yes & Yes & $\begin{array}{l}\text { Yes } \\
\text { Baseline data from an RCT on } \\
\text { behavioural intervention }\end{array}$ & $\begin{array}{l}\text { Comparable to US diabetes } \\
\text { population- } 78 \% \text { Caucasian; } 84 \% \\
\text { married, } 76 \% \text { income }>\$ 50,000\end{array}$ & No \\
\hline $\begin{array}{l}\text { 16.Johnson } \\
(2013)\end{array}$ & $\begin{array}{l}\text { Yes } \\
\text { Evaluate FOH, hypoglycaemia } \\
\text { and quality of life }\end{array}$ & Yes & Yes & $\begin{array}{l}\text { Yes } \\
\text { Time commitment } \\
\text { Non-responders were younger and } \\
\text { had shorter DM duration }\end{array}$ & $\begin{array}{l}\text { Representative of clinic population } \\
\text { but few parental demographics } \\
\text { provided }\end{array}$ & No \\
\hline $\begin{array}{l}\text { 17.Kamps } \\
(2005)\end{array}$ & $\begin{array}{l}\text { Yes } \\
\text { Provision of preliminary } \\
\text { psychometric data on } \mathrm{CHI}\end{array}$ & Yes & Yes & $\begin{array}{l}\text { Yes } \\
\text { Recruitment from within a summer } \\
\text { camp }\end{array}$ & $\begin{array}{l}\text { Less so- } 87 \% \text { Caucasian, summer } \\
\text { camp attendees, limited other } \\
\text { information }\end{array}$ & No \\
\hline $\begin{array}{l}\text { 18.Markowitz } \\
\text { (2012) }\end{array}$ & $\begin{array}{l}\text { Yes } \\
\text { Comparison of psychological } \\
\text { characteristics CGM v. SMBG }\end{array}$ & Yes & Yes & $\begin{array}{l}\text { Yes } \\
\text { One site data from those already } \\
\text { recruited to larger JDRF-CGM trial }\end{array}$ & $\begin{array}{l}\text { Unclear } \\
\text { Limited demographics included }\end{array}$ & No \\
\hline $\begin{array}{l}\text { 19.Mitchell } \\
(2009)\end{array}$ & $\begin{array}{l}\text { Yes } \\
\text { Correlates of fathers parenting } \\
\text { stress including FoH }\end{array}$ & Yes & Yes & $\begin{array}{l}\text { Perhaps } \\
\text { Questionnaires time commitment } \\
\$ 10 \text { gift card incentive } \\
\text { Participants from larger study }\end{array}$ & $\begin{array}{l}\text { Reflective of Atlanta population } \\
\text { Only fathers explored, } 96 \% \\
\text { married, } 78 \% \text { income }>\$ 75,000\end{array}$ & No \\
\hline $\begin{array}{l}\text { 20.Pate } \\
\text { (2019) }\end{array}$ & $\begin{array}{l}\text { Yes } \\
\text { Parental FoH, anxiety and } \\
\text { well-being }\end{array}$ & Yes & Yes & $\begin{array}{l}\text { Perhaps } \\
\text { Questionnaires time commitment }\end{array}$ & $\begin{array}{l}\text { Partially } \\
\text { Majority married \& employed } \\
\text { Sample HbA1c significantly lower }\end{array}$ & No \\
\hline $\begin{array}{l}\text { 21.Patton } \\
\text { (2007) }\end{array}$ & $\begin{array}{l}\text { Yes } \\
\text { Parental FoH \& BG levels }\end{array}$ & Yes & Yes & $\begin{array}{l}\text { Perhaps } \\
2 \text { weeks of SMBG } 4 \text { times/day } \\
\$ 20 \text { gift card incentive }\end{array}$ & $\begin{array}{l}\text { Representative of clinic population } \\
96 \% \text { white; } 92 \% \text { married } \\
96 \%>\text { SES class III }\end{array}$ & No \\
\hline $\begin{array}{l}\text { 22.Patton } \\
\text { (2008) }\end{array}$ & $\begin{array}{l}\text { Yes } \\
\text { Development of HFS-PYC }\end{array}$ & Yes & Yes & $\begin{array}{l}\text { Perhaps } \\
2 \text { weeks SMBG } 4 \text { times/day } \\
\$ 20 \text { gift card incentive }\end{array}$ & $\begin{array}{l}\text { Representative of clinic population } \\
72 \% \text { white; } 74 \% \text { married } \\
85 \%>\text { SES class III }\end{array}$ & No \\
\hline $\begin{array}{l}\text { 23.Patton } \\
\text { (2011) }\end{array}$ & $\begin{array}{l}\text { Yes } \\
\text { Parenting stress \& FoH/ } \\
\text { depression }\end{array}$ & Yes & Yes & $\begin{array}{l}\text { Perhaps } \\
\$ 50 \text { reimbursement } \\
\text { English must be spoken at home }\end{array}$ & $\begin{array}{l}\text { Representative of clinic population } \\
82 \% \text { white; } 74 \% \text { married } \\
54 \% \text { income }>\$ 50,000\end{array}$ & No \\
\hline $\begin{array}{l}\text { 24.Patton } \\
\text { (2017) }\end{array}$ & $\begin{array}{l}\text { Yes } \\
\text { Update psychometric } \\
\text { properties of HFS-PYC }\end{array}$ & Yes & Yes & NA & $\begin{array}{l}\text { Representative of clinic population } \\
91 \% \text { white }\end{array}$ & No \\
\hline $\begin{array}{l}\text { 25.Shepard } \\
(2014)\end{array}$ & $\begin{array}{l}\text { Yes } \\
\text { Exploring constructs of HFS-P } \\
\text { and HFS-C }\end{array}$ & Yes & Yes & $\begin{array}{l}\text { Perhaps } \\
4 \text { weeks SMBG } 4 \text { times/day }\end{array}$ & $\begin{array}{l}\text { Representative of clinic population } \\
93 \% \text { Caucasian, } 4 \% \text { African } \\
87 \% \text { married } \\
\text { Better glycaemic control }\end{array}$ & No \\
\hline $\begin{array}{l}\text { 26.Streisand } \\
(2005)\end{array}$ & $\begin{array}{l}\text { Yes } \\
\text { Parenting stress and its } \\
\text { correlates (including FoH) }\end{array}$ & Yes & Yes & $\begin{array}{l}\text { Yes - participants consented to } \\
\text { longitudinal study } \\
\$ 25 \text { reimbursement }\end{array}$ & $\begin{array}{l}\text { Yes } \\
79 \% \text { Caucasian; } 84 \% \text { married } \\
46 \% \text { SES class III }\end{array}$ & No \\
\hline $\begin{array}{l}\text { 27.Van Name } \\
\text { (2017) }\end{array}$ & $\begin{array}{l}\text { Yes } \\
\text { FoH in parents of young } \\
\text { children }\end{array}$ & Yes & Yes & $\begin{array}{l}\text { Perhaps } \\
\text { Questionnaire time commitment }\end{array}$ & $\begin{array}{l}\text { Yes } \\
77 \% \text { white } 10 \% \text { Hispanic } 6 \% \text { black } \\
\text { But } 52 \% \text { income }>\$ 75,000\end{array}$ & No \\
\hline $\begin{array}{l}\text { 28.Viaene } \\
\text { (2017) }\end{array}$ & $\begin{array}{l}\text { Yes } \\
\text { Parenting stress, FoH and } \\
\text { metabolic control }\end{array}$ & Yes & Yes & $\begin{array}{l}\text { Perhaps } \\
\text { Opportunistic recruitment from } \\
\text { clinic attendance; Dutch-speaking }\end{array}$ & $\begin{array}{l}\text { Perhaps } \\
76 \% \text { married; limited other } \\
\text { demographic information available }\end{array}$ & No \\
\hline
\end{tabular}


Appendix 2. Quality Assessment Tables (continued)

\begin{tabular}{|c|c|c|c|c|c|c|}
\hline Study & 7 & 8 & 9 & 10 & 11 & 12 \\
\hline $\begin{array}{l}\text { Authors } \\
\text { Year }\end{array}$ & $\begin{array}{l}\text { Satisfactory } \\
\text { response } \\
\text { rate }\end{array}$ & $\begin{array}{l}\text { Valid \& reliable measures } \\
\text { (Cronbach's alpha= a) }\end{array}$ & $\begin{array}{l}\text { Statistical } \\
\text { significance } \\
\text { assessed }\end{array}$ & $\begin{array}{l}\text { Confidence } \\
\text { intervals } \\
\text { given }\end{array}$ & $\begin{array}{l}\text { Confounders } \\
\text { accounted for }\end{array}$ & Results applicable locally \\
\hline $\begin{array}{l}\text { 1. Aalders } \\
\text { (2018) }\end{array}$ & Yes- $79 \%$ & $\begin{array}{l}\text { Yes- HFS-P worry scale }(a=0.88) \\
\text { Freiburg Mindfulness Inventory }(a=0.83) \\
\text { Interpersonal Mindfulness in Parenting Scale }(a=0.85)\end{array}$ & Yes & No & Yes & $\begin{array}{l}\text { Partially } \\
\text { Similar demographics but higher } \\
\text { SES and married state; higher CSII }\end{array}$ \\
\hline $\begin{array}{l}\text { 2. Al Hayek } \\
(2015)\end{array}$ & Unknown & $\begin{array}{l}\text { Yes- HFS-C }(a=0.86) \text { Arabic translated } \\
\text { Screen for Child Anxiety-Related Disorders }(a=0.91) \\
\text { Socio-demographic/ clinical questionnaire }\end{array}$ & Yes & Yes & Yes & Less so- Saudi Arabia based \\
\hline $\begin{array}{l}\text { 3. Amiri } \\
\text { (2014) }\end{array}$ & Yes- $81 \%$ & $\begin{array}{l}\text { Yes- HFS-C }(a=0.89) \text { Persian translated } \\
\text { Self-Efficacy for Diabetes Scale- Child Version (SED-C) } \\
\text { Diabetes History Questionnaire }\end{array}$ & Yes & Yes & Yes & $\begin{array}{l}\text { Less so - Iran based } \\
\text { Different cultural environment- only } \\
25 \% \text { mothers employed; all MDI }\end{array}$ \\
\hline $\begin{array}{l}\text { 4. Amiri } \\
\text { (2018) }\end{array}$ & $\begin{array}{l}\text { Yes- } 81 \% \\
\text { Same data } 2014\end{array}$ & $\begin{array}{l}\text { Yes- HFS-P }(a=0.94) \text { Persian translated } \\
\text { SED-P }(a=0.84) \\
\text { Paediatric Inventory for Parents (PIP) }(a=0.95-6)\end{array}$ & Yes & No & Yes & $\begin{array}{l}\text { Less so - Iran based } \\
\text { Different cultural environment- only } \\
25 \% \text { mothers employed; all MDI }\end{array}$ \\
\hline $\begin{array}{l}\text { 5. Di Battista } \\
\text { (2009) }\end{array}$ & $\begin{array}{l}\text { No- } 23 \% \text { US; } \\
45 \% \text { Canada }\end{array}$ & $\begin{array}{l}\text { Yes- HFS-P }(a=0.87) \\
\text { Self-report demographics/HbA1c; Diabetes QoL Measure } \\
\text { Social Anxiety Scale for Adolescents } \\
\text { Summary of Diabetes Self-Care Regimen }\end{array}$ & Yes & No & Yes & $\begin{array}{l}\text { Partially } \\
\text { Small North American sample with } \\
\text { poor response rate }\end{array}$ \\
\hline $\begin{array}{l}\text { 6. Forsander } \\
\text { (2016) }\end{array}$ & $\begin{array}{l}\text { No- } 21 \% \\
\text { But large sample }\end{array}$ & $\begin{array}{l}\text { No- scale of } 1 \text { to } 10 \text { to assess FoH } \\
\text { Diabetes Distress Scale }\end{array}$ & Yes & Yes & Yes & $\begin{array}{l}\text { Partially } \\
\text { Large European sample } \\
\text { Poor response rate; high SES }\end{array}$ \\
\hline $\begin{array}{l}\text { 7. Freckleton } \\
\text { (2014) }\end{array}$ & Yes- $62 \%$ & $\begin{array}{l}\text { Yes- HFS }(a=0.86) \\
\text { Illness Perception Questionnaire } \\
\text { 7-day diabetes diary management }\end{array}$ & Yes & No & Yes & $\begin{array}{l}\text { Partially } \\
\text { All mothers and all children MDI } \\
\text { users }\end{array}$ \\
\hline $\begin{array}{l}\text { 8. Frederick } \\
\text { (2011) }\end{array}$ & Unknown & $\begin{array}{l}\text { Yes- CHI }(a=0.89) \\
\text { Diabetes Behaviour Rating Scale (DRBS) }\end{array}$ & Yes & Yes & Yes & $\begin{array}{l}\text { Partially } \\
\text { Diverse sample, decent size } \\
\text { No SES data }\end{array}$ \\
\hline $\begin{array}{l}\text { 9. Gonder-Frederick } \\
\text { (2006) }\end{array}$ & Yes- $63 \%$ & $\begin{array}{l}\text { Yes- HFS-P }(a=0.89) \& \text { HFS-C }(a=0.86) \\
\text { State Trait Personality Inventory (STPI) } \\
\text { State Trait Anxiety Inventory for Children (STAIC) } \\
\text { Diabetes specific questionnaire }\end{array}$ & Yes & No & Yes & $\begin{array}{l}\text { Partially } \\
\text { Only one father included } \\
\text { Limited SES diversity }\end{array}$ \\
\hline $\begin{array}{l}\text { 10.Gonder-Frederick } \\
\text { (2011) }\end{array}$ & $\begin{array}{l}\text { NA } \\
\text { Aggregated data }\end{array}$ & $\begin{array}{l}\text { Yes- HFS-P }(a=0.86) \& \text { HFS-C }(a=0.85) \\
\text { STPI } \\
\text { STAIC }\end{array}$ & Yes & No & Yes & $\begin{array}{l}\text { Yes } \\
\text { Good sample size and variance } \\
\text { But aggregated data over a decade }\end{array}$ \\
\hline $\begin{aligned} \text { 11.Grey } \\
(2009)\end{aligned}$ & No- $40 \%$ & $\begin{array}{l}\text { Yes- HFS, STAI a }=0.93 \\
\text { Center for Epidemiological Studies Depression Scale } \\
\text { Issues in Coping with IDDM-Parent scale }(a=0.87-88)\end{array}$ & Yes & No & Yes & $\begin{array}{l}\text { Partially } \\
\text { Only mothers included }\end{array}$ \\
\hline $\begin{array}{l}\text { 12.Haugstvedt } \\
\text { (2010) }\end{array}$ & Yes- $71 \%$ & $\begin{array}{l}\text { Yes- HFS-P }(a=0.87-0.94) \\
\text { Demographic questionnaire } \\
\text { Hopkins Symptom Checklist-25 (HSCL-25) }(a=0.87-92)\end{array}$ & Yes & Yes & Yes & $\begin{array}{l}\text { Partially } \\
\text { Decent sample size and } \\
\text { comparable with UK }\end{array}$ \\
\hline $\begin{array}{l}\text { 13. Haugstvedt } \\
\text { (2015) }\end{array}$ & $\begin{array}{l}\text { Yes- } 71 \% \\
\text { Same data } 2010\end{array}$ & $\begin{array}{l}\text { Yes- HFS-P } \\
\text { HSCL-25 }\end{array}$ & Yes & No & Yes & $\begin{array}{l}\text { Partially } \\
\text { Decent sample size and } \\
\text { comparable with UK }\end{array}$ \\
\hline $\begin{array}{l}\text { 14. Hawkes } \\
\text { (2014) }\end{array}$ & Unknown & $\begin{array}{l}\text { HFS-PYC } \\
\text { Demographic questionnaire \& self-report hypoglycaemia }\end{array}$ & Yes & No & Unclear & $\begin{array}{l}\text { Perhaps } \\
\text { Limited parental demographics } \\
\text { Irish population similar to UK }\end{array}$ \\
\hline $\begin{array}{l}\text { 15. Herbert } \\
(2014)\end{array}$ & No- $47 \%$ & $\begin{array}{l}\text { Yes- HFS-PYC }(\mathrm{a}=0.92) \\
\text { Pittsburgh Sleep Quality Index (PSQI); SED-P ( } \mathrm{a}=0.78) \\
\text { Demographic \& medical questionnaires } \\
24 \mathrm{~h} \text { recall interview of diabetes tasks + glucometer data }\end{array}$ & Yes & No & Yes & $\begin{array}{l}\text { Yes } \\
\text { Relatively diverse US population }\end{array}$ \\
\hline $\begin{array}{l}\text { 16.Johnson } \\
\text { (2013) }\end{array}$ & $\begin{array}{l}\text { No- } 49 \% \\
\text { But large sample }\end{array}$ & $\begin{array}{l}\text { Yes- HFS } \\
\text { PedsQL Diabetes Module } \\
\text { Clarke's hypoglycaemia awareness questionnaire } \\
\text { Clinical data from W Australia Childhood Diabetes Database }\end{array}$ & Yes & No & No & $\begin{array}{l}\text { Yes } \\
\text { Large sample; similar CSII use } \\
\text { But no parental demographics }\end{array}$ \\
\hline $\begin{array}{c}\text { 17.Kamps } \\
\text { (2005) }\end{array}$ & Yes- $65 \%$ & $\begin{array}{l}\text { Yes- HFS-C and CHI } \\
\text { RCMAS } \\
\text { Hypoglycaemia History Form }\end{array}$ & Yes & No & Unclear & $\begin{array}{l}\text { Partially } \\
\text { High SES in sample } \\
\text { Summer camp attendants }\end{array}$ \\
\hline $\begin{array}{l}\text { 18.Markowitz } \\
\text { (2012) }\end{array}$ & $\begin{array}{l}\text { Yes- } 96 \% \\
\text { Of participants } \\
\text { already recruited } \\
\text { to JDRF-CGM } \\
\text { trial }\end{array}$ & $\begin{array}{l}\text { Yes- HFS } \\
\text { Pediatric QOL Inventory; Short Form Health Survey } \\
\text { Center for Epidemiologic Studies Depression scale (CES-D) } \\
\text { BGM Communication Questionnaire Diabetes Family Conflict } \\
\text { Scale (DFCS); STAI \& PAID; Children's Depression Inventory }\end{array}$ & Yes & No & Yes & $\begin{array}{l}\text { Partially } \\
\text { Very small study but UK based } \\
\text { Limited demographics included } \\
\text { Included adult participants but } \\
\text { result separated by age group }\end{array}$ \\
\hline $\begin{array}{l}\text { 19.Mitchell } \\
\text { (2009) }\end{array}$ & Yes- $86 \%$ & $\begin{array}{l}\text { Yes- HFS-P }(a=0.92) \text {, PIP }(a=0.95) \text {; recall interview } \\
\text { Eyberg Child Behaviour Inventory } \\
\text { Self-Efficacy for Diabetes Scale (SED) } \\
\text { STAI }(a=0.93) ; \text { Hope Scale }(a=0.79)\end{array}$ & Yes & No & Yes & $\begin{array}{l}\text { Partially } \\
\text { Only fathers; high income/ married } \\
\text { Not generalisable to single fathers }\end{array}$ \\
\hline $\begin{array}{l}\text { 20.Pate } \\
(2019)\end{array}$ & Yes- $62 \%$ & $\begin{array}{l}\text { Yes- HFS-P }(a=0.89), \text { STAI }(a=0.90) \\
\text { Positive and Negative Affect Schedule (PANAS) } \\
\text { Satisfaction with Life Scale (SWLS) }(a=0.87)\end{array}$ & Yes & No & Yes & $\begin{array}{l}\text { Partially } \\
\text { Reliance on translated HFS } \\
\text { Sample HbA1c significantly lower }\end{array}$ \\
\hline $\begin{array}{c}\text { 21.Patton } \\
\text { (2007) }\end{array}$ & Yes- $86 \%$ & $\begin{array}{l}\text { Yes - HFS-PYC }(a=0.86) \\
\text { SMBG + HbA1c } \\
\text { Self-report demographic \& hypoglycaemia history }\end{array}$ & Yes & No & No & $\begin{array}{l}\text { Partially } \\
\text { Small sample \& limited diversity } \\
\text { Single clinic Cincinnati } \\
100 \% \text { CSII users }\end{array}$ \\
\hline $\begin{array}{l}\text { 22.Patton } \\
\text { (2008) }\end{array}$ & Yes- $73 \%$ & $\begin{array}{l}\text { Yes - HFS-PYC }(a=0.91) \\
\text { SMBG + HbA1c } \\
\text { Self-report demographic \& hypoglycaemia history }\end{array}$ & Yes & No & No & $\begin{array}{l}\text { Partially } \\
\text { Limited diversity } \\
\text { Single clinic Cincinnati } \\
\text { Majority had HbA1c } 7.5-8.5 \%\end{array}$ \\
\hline $\begin{array}{l}\text { 23.Patton } \\
\text { (2011) }\end{array}$ & Just- $51 \%$ & $\begin{array}{l}\text { Yes- HFS-PYC } \\
\text { Behavioural Pediatric Feeding Assessment Scale } \\
\text { Pediatric Inventory for Parents (PIP) } \\
\text { Beck Depression Inventory-Second Edition (BDI-II) }\end{array}$ & Yes & No & Yes & $\begin{array}{l}\text { Partially } \\
\text { Small homogenous sample } \\
2 \text { clinics in the Midwest } \\
\text { High CSII use; majority mothers }\end{array}$ \\
\hline $\begin{array}{l}\text { 24.Patton } \\
\text { (2017) }\end{array}$ & $\begin{array}{l}\text { NA } \\
3 \text { datasets }\end{array}$ & Yes- HFS-PYC & Yes & No & Unclear & $\begin{array}{l}\text { Partially } \\
3 \text { datasets over } 5 \text { years } \\
\text { Homogenous sample } \\
100 \% \text { CSII users }\end{array}$ \\
\hline $\begin{array}{l}\text { 25.Shepard } \\
\text { (2014) }\end{array}$ & $\begin{array}{l}\text { NA } \\
5 \text { studies }\end{array}$ & $\begin{array}{l}\text { Yes- HFS-C and HFS-P } \\
\text { STPI }(a=0.8-87), \text { STAIC } \\
\text { Self-report demographics \& hypoglycaemic history }\end{array}$ & Yes & No & Yes & $\begin{array}{l}\text { Partially } \\
5 \text { studies data over } 10 \text { years } \\
\text { All from same Virginia lab } \\
\text { Narrow demographics }\end{array}$ \\
\hline $\begin{array}{l}\text { 26.Streisand } \\
(2005)\end{array}$ & Yes $-80 \%$ & $\begin{array}{l}\text { Yes- HFS-P }(a=0.90) \\
\text { Diabetes Family Responsibility Questionnaire (DFRQ) } \\
\text { PIP }(a=0.94) ; \text { SED }(a=0.87) \\
\text { Demographic and Medical History Questionnaire }\end{array}$ & Yes & No & Yes & $\begin{array}{l}\text { Yes } \\
\text { But note wide child age range } \\
\text { Majority mothers }\end{array}$ \\
\hline $\begin{array}{l}\text { 27.Van Name } \\
(2017)\end{array}$ & $\begin{array}{l}\text { Yes- } 71 \% \text { at site } \\
\text { level } \\
\text { Unclear at } \\
\text { individual level }\end{array}$ & $\begin{array}{l}\text { Yes- HFS-P Worry scale } \\
\text { Self-report demographics, DKA \& SH }\end{array}$ & Yes & No & Yes & $\begin{array}{l}\text { Yes } \\
\text { Large registry T1DM exchange } \\
\text { Diverse sample but high SES } \\
\text { High CGM use }(32 \%) \\
\text { No parent gender identification }\end{array}$ \\
\hline $\begin{array}{c}\text { 28.Viaene } \\
\text { (2017) }\end{array}$ & Yes- $74 \%$ & $\begin{array}{l}\text { Yes- HFS-P }(a=0.86) ; \text { HFS-C }(a=0.68) \\
\text { Nijmegen Parenting Stress Index- Short form (NPSI-S) }\end{array}$ & Yes & Yes & Yes & $\begin{array}{l}\text { Yes } \\
\text { But small dataset a } \\
\text { Limited demographics available }\end{array}$ \\
\hline
\end{tabular}


Appendix 2. Quality Assessment Tables (continued)

B. Randomised Controlled Trials $(n=8)$

CASP Randomised Control Trial Checklist (CASP, 2018)

1. Did the trial address a clearly focused issue?

2. Was the assignment of patients to treatments randomised?

3. Were all of the patients who entered the trial properly accounted for at its conclusion?

4. Were patients, health workers and study personnel 'blind' to treatment'?

5. Were the groups similar at the start of the trial?

6. Aside from the experimental intervention, were the groups treated equally?

7. How large was the treatment effect?

8. How precise was the estimate of the treatment effect?

9. Can the results be applied to the local population, or in your context?

10. Were all clinically important outcomes considered?

11. Are the benefits worth the harms and costs? 
Appendix 2. Quality Assessment Tables (continued)

\begin{tabular}{|c|c|c|c|c|c|c|}
\hline & 1 & 2 & 3 & 4 & 5 & 6 \\
\hline $\begin{array}{l}\text { First Author } \\
\text { Publication Year }\end{array}$ & $\begin{array}{l}\text { Clearly } \\
\text { focused } \\
\text { issue }\end{array}$ & $\begin{array}{l}\text { Assignment } \\
\text { randomised }\end{array}$ & Patient accountability & Blinded intervention & $\begin{array}{l}\text { Similar baseline } \\
\text { characteristics }\end{array}$ & $\begin{array}{l}\text { Equal treatment of } \\
\text { two groups }\end{array}$ \\
\hline $\begin{array}{l}\text { 1. Abraham } \\
\text { (2018) }\end{array}$ & $\begin{array}{l}\text { Yes } \\
\text { PLGM v. SAPT }\end{array}$ & $\begin{array}{l}\text { Yes } \\
\text { Minimisation at randomisation }\end{array}$ & $\begin{array}{l}\text { Yes- consort diagram } \\
19 \% \text { loss (withdrawal/ deviation) }\end{array}$ & No & Yes & Yes \\
\hline $\begin{array}{l}\text { 2. Barnard } \\
(2014)\end{array}$ & $\begin{array}{l}\text { Yes } \\
\text { CLS v. SAPT }\end{array}$ & $\begin{array}{l}\text { Yes } \\
\text { Permuted block-four approach }\end{array}$ & $\begin{array}{l}\text { Yes } \\
52 \% \text { recruitment; } 1 \text { withdrawal }\end{array}$ & $\begin{array}{l}\text { Not to patient } \\
\text { Allocation concealed to staff }\end{array}$ & Unclear & $\begin{array}{l}\text { Almost } \\
\text { CLS- extra supervision }\end{array}$ \\
\hline $\begin{array}{l}\text { 3. Burkhardt } \\
\text { (2018) }\end{array}$ & $\begin{array}{l}\text { Yes } \\
\text { CGM v. SMBG }\end{array}$ & $\begin{array}{l}\text { Yes } \\
\text { Computer generated }\end{array}$ & No & No & Unclear & Yes \\
\hline $\begin{array}{l}\text { 4. JDRF CGM } \\
(2010)\end{array}$ & $\begin{array}{l}\text { Yes } \\
\text { CGM v. SMBG }\end{array}$ & $\begin{array}{l}\text { Yes } \\
\text { Permuted block design } \\
\text { Stratified- centre, age,HbA1c }\end{array}$ & $\begin{array}{l}\text { Yes } \\
95-100 \% \text { completion rate }\end{array}$ & No & Yes & $\begin{array}{l}\text { Almost } \\
\text { CGM- additional direction }\end{array}$ \\
\hline $\begin{array}{l}\text { 5. Mayer-Davis } \\
(2018)\end{array}$ & $\begin{array}{l}\text { Yes } \\
\text { FLEX v. control }\end{array}$ & $\begin{array}{l}\text { Yes } \\
\text { Automated block method } \\
\text { Stratified by site \& HbA1c }\end{array}$ & $\begin{array}{l}\text { Yes- consort diagram } \\
16.5 \% \text { ineligible; } 51 \% \text { refused } \\
\text { Final sample- } 93 \% \text { retention rate }\end{array}$ & $\begin{array}{l}\text { Not to patient } \\
\text { Allocation concealed to staff }\end{array}$ & Yes & Yes \\
\hline $\begin{array}{l}\text { 6.Mueller-Godeffroy } \\
\text { (2018) }\end{array}$ & $\begin{array}{l}\text { Yes } \\
\text { CSII v. MDI }\end{array}$ & $\begin{array}{l}\text { Yes } \\
\text { Software; stratified by centre }\end{array}$ & $\begin{array}{l}\text { Yes- consort diagram } \\
57 \% \text { recruitment } \\
15 \% \text { loss to follow up }\end{array}$ & $\begin{array}{l}\text { Not to patient } \\
\text { Allocation concealed to staff }\end{array}$ & Yes & Yes \\
\hline $\begin{array}{l}\text { 7. Patton } \\
\text { (2019) }\end{array}$ & $\begin{array}{l}\text { Yes } \\
\text { RECHiP v. } \\
\text { control }\end{array}$ & $\begin{array}{l}\text { Yes } \\
\text { Block assignment by child sex }\end{array}$ & $\begin{array}{l}\text { Yes- consort diagram } \\
32 \% \text { recruitment } \\
16 \% \text { excluded final analysis }\end{array}$ & No & Unclear & Yes \\
\hline $\begin{array}{l}\text { 8. Ziegler } \\
\text { (2015) }\end{array}$ & $\begin{array}{l}\text { Yes } \\
\text { AP v. SAPT }\end{array}$ & $\begin{array}{l}\text { Yes } \\
\text { Computer software blocked } \\
\text { randomisation }\end{array}$ & No & No & Unclear & Unclear \\
\hline
\end{tabular}

\begin{tabular}{|c|c|c|c|c|c|}
\hline Study & 7 & 8 & 9 & 10 & 11 \\
\hline $\begin{array}{l}\text { First Author } \\
\text { Publication Year }\end{array}$ & Treatment Effect & Precision Estimate & $\begin{array}{l}\text { Applicable } \\
\text { Results }\end{array}$ & $\begin{array}{l}\text { Significant } \\
\text { outcomes } \\
\text { explored }\end{array}$ & $\begin{array}{l}\text { Benefits outweigh } \\
\text { harms/ costs }\end{array}$ \\
\hline $\begin{array}{l}\text { 1. Abraham } \\
\text { (2018) }\end{array}$ & $\begin{array}{l}\text { Primary outcome: time spent in hypoglycaemia } \\
\text { PGLM hypo reduction from } 2.8 \% \text { to } 1.4 \% \\
\text { No differences for HbA1c, HFS and Peds QL } \\
\text { scores }\end{array}$ & $\begin{array}{l}\text { Reduction more with PGLM v. SAPT }(p<0.0001 \text { ) } \\
\text { (mean difference }-0.95 \%[95 \% \mathrm{Cl}-1.30 \text { to }-0.61)\end{array}$ & $\begin{array}{l}\text { Somewhat } \\
5 \text { Australian centres } \\
\text { Limited demographics }\end{array}$ & Yes & $\begin{array}{l}\text { No adverse events } \\
\text { Cost not explored }\end{array}$ \\
\hline $\begin{array}{l}\text { 2. Barnard } \\
\text { (2014) }\end{array}$ & $\begin{array}{l}\text { Primary outcome: time spent in target BG range } \\
\text { Nocturnal hypo less in CLS than CGM } 10 \text { v. } 17 \% \\
\text { Mixed results for HFS scores }\end{array}$ & $\begin{array}{l}\text { Time spent in target increased from } 47 \% \text { to } 64 \% \\
\text { with CLS ( }<<0.001) \\
\text { HFS changes were not statistically significant }\end{array}$ & $\begin{array}{l}\text { Yes- UK based trial } \\
2 \text { units; home trial } \\
\text { But very small sample }\end{array}$ & Yes & $\begin{array}{l}\text { No adverse events } \\
\text { Cost not explored }\end{array}$ \\
\hline $\begin{array}{l}\text { 3. Burkhardt } \\
\text { (2018) }\end{array}$ & $\begin{array}{l}\text { Primary outcome: parental FoH on HFS } \\
\text { HFS-P lower after intervention: } 54.9 \text { v. } 44.7\end{array}$ & $\begin{array}{l}\text { Least square mean difference control v CGM: } \\
-8.5(95 \% \mathrm{Cl}-12.7 \text { to }-4.4 ; p<0.001)\end{array}$ & $\begin{array}{l}\text { Somewhat } \\
\text { Australian study- small } \\
\text { Limited demographics }\end{array}$ & Yes & $\begin{array}{l}\text { No adverse events } \\
\text { Cost not explored }\end{array}$ \\
\hline $\begin{array}{l}\text { 4. JDRF CGM } \\
\text { Study Group } \\
\text { (2010) }\end{array}$ & $\begin{array}{l}\text { Primary outcome: HbA1c - no significant } \\
\text { difference for CYP; for HFS scores slight } \\
\text { improvement in CGM group >18y }(<0.05)\end{array}$ & No significant HFS changes in youth or parents & $\begin{array}{l}\text { Yes- } 10 \text { UK centres } \\
\text { Included adults } \\
\text { Limited diversity }\end{array}$ & Yes & $\begin{array}{l}5-10 \% \text { at least one SH } \\
\text { No difference in groups } \\
\text { Appears cost-effective }\end{array}$ \\
\hline $\begin{array}{l}\text { 5. Mayer-Davis } \\
\text { (2018) }\end{array}$ & $\begin{array}{l}\text { Primary outcome: HbA1c; no effect } \\
\text { All domains of HFS decreased with intervention }\end{array}$ & $\begin{array}{l}\text { Only significant HFS reductions in behaviour to } \\
\text { maintain high BG in parents }(p=0.005) \text { and } \\
\text { worry/ helplessness in adolescents }(p=0.04)\end{array}$ & $\begin{array}{l}\text { Somewhat } \\
\text { Diverse US sample }\end{array}$ & Yes & $\begin{array}{l}34 \text { adverse events } \\
\text { None study related } \\
\text { Cost not explored }\end{array}$ \\
\hline $\begin{array}{l}\text { 6.Mueller-Godeffroy } \\
\text { (2018) }\end{array}$ & Significant improvement in parental HFS & $\begin{array}{l}\text { Primary outcome DHRQOL significantly better } \\
\text { for CSII (MD } 5.95,1.19-01.71 \mathrm{p}=0.016 \text { ) }\end{array}$ & $\begin{array}{l}\text { Yes } \\
\text { Multiple centres } \\
\text { Germany }\end{array}$ & Yes & $\begin{array}{l}\text { No adverse events } \\
\text { Cost not explored }\end{array}$ \\
\hline $\begin{array}{l}\text { 7. Patton } \\
\text { (2019) }\end{array}$ & $\begin{array}{l}\text { Primary outcome: HFS + PIP score } \\
\text { Significant reduction in HFS }(p=0.04) \text { and in } \\
\text { parenting stress frequency } p=0.092)\end{array}$ & $\begin{array}{l}\text { Parental HFS-PYC total score } 71.5 \text { to } 59.9 \\
(6.53-16.61 ; p<0.001)\end{array}$ & $\begin{array}{l}\text { A little } \\
\text { Small US sample } \\
\text { Homogenous }\end{array}$ & Yes & $\begin{array}{l}59.5 \% \text { hypo } 1-2 / \text { week } \\
\text { Maintenance only at } 3 \mathrm{~m} \\
\text { Cost not explored }\end{array}$ \\
\hline $\begin{array}{l}\text { 8. Ziegler } \\
\text { (2015) }\end{array}$ & $\begin{array}{l}\text { After } 4 \text { nights on the AP system HFS worry } \\
\text { decreased significantly }(p=0.017)\end{array}$ & $\begin{array}{l}\text { Significant change only in HFS-W not HFS-B } \\
\text { Too short a duration of intervention }\end{array}$ & $\begin{array}{l}\text { Multinational } \\
\text { Limited demographics } \\
19 \text { adults included }\end{array}$ & Yes & Cost not explored \\
\hline
\end{tabular}


Appendix 2. Quality Assessment Tables (continued)

\section{Pre-Post Prospective Studies $(n=5)$}

NIH Quality Assessment Tool for Before-After (Pre-Post) Studies (NIH, 2018)

1. Was the study question or objective clearly stated?

2. Were eligibility/ selection criteria for the study population prespecified and clearly described?

3. Were the participants in the study representative of those who would be eligible for the intervention in the general/clinical population of interest?

4. Were all eligible participants that met the prespecified entry criteria enrolled?

5. Was the sample size sufficiently large to provide confidence in the findings?

6. Was the test/ service/ intervention clearly described and delivered consistently across the study population?

7. Were the outcome measures prespecified, clearly defined, valid, reliable, and assessed consistently across all study participants

8. Were the people assessing the outcomes blinded to the participants' exposures/ interventions?

9. Was the loss to follow-up after baseline $20 \%$ or less? Were those lost to follow-up accounted for in the analysis?

10. Did the statistical methods examine changes in outcome measures from before to after the intervention and provide $p$ values?

11. Were outcome measures of interest taken multiple times before the intervention and multiple times after the intervention (i.e., did they use an interrupted time-series design?

12. If the intervention was conducted at a group level (e.g., a whole hospital, a community, etc) did the statistical analysis take into account the use of individuallevel data to determine effects at the group level?

\begin{tabular}{|c|c|c|c|c|c|c|}
\hline Study & 1 & 2 & 3 & 4 & 5 & 6 \\
\hline $\begin{array}{l}\text { First Author } \\
\text { Publication Year }\end{array}$ & $\begin{array}{l}\text { Clearly stated } \\
\text { objective }\end{array}$ & $\begin{array}{l}\text { Clearly described } \\
\text { eligibility criteria }\end{array}$ & $\begin{array}{l}\text { Study participants } \\
\text { representative }\end{array}$ & $\begin{array}{l}\text { All eligible } \\
\text { participants } \\
\text { enrolled }\end{array}$ & $\begin{array}{l}\text { Sample size } \\
\text { sufficient }\end{array}$ & $\begin{array}{l}\text { Intervention } \\
\text { clear and } \\
\text { consistent }\end{array}$ \\
\hline $\begin{array}{l}\text { 1.Al Hayek } \\
(2017)\end{array}$ & $\begin{array}{l}\text { Yes }(3 m) \\
\text { FGM- FoH/ QoL } \\
\text { HbA1c }\end{array}$ & $\begin{array}{l}\text { Yes: } 13-19 \text { years } \\
\text { Minimum } 6 \mathrm{~m} \text { T1DM } \\
\text { No recent SH/ DKA }\end{array}$ & $\begin{array}{l}\text { Yes- of Saudi Arabia } \\
\text { Limited demographics }\end{array}$ & Unclear & $\begin{array}{l}\text { No }(n=47) \\
\text { No power calculation }\end{array}$ & Yes \\
\hline $\begin{array}{l}\text { 2. Cai } \\
(2017)\end{array}$ & $\begin{array}{l}\text { Yes }(3 m) \\
\text { Workshop- FoH/QoL } \\
\text { HbA1c }\end{array}$ & $\begin{array}{l}\text { Yes: } 8-16 \text { years } \\
\text { Minimum 6m T1DM } \\
\text { No co-morbidity }\end{array}$ & $\begin{array}{l}\text { Yes- of UK clinic population } \\
\text { Ethnically diverse }\end{array}$ & $\begin{array}{l}\text { No- pilot study } \\
\text { Only } 89 \text { of } 300- \\
33 \% \text { recruitment }\end{array}$ & $\begin{array}{l}\text { Almost }(n=22) \\
\text { Aimed } 32 \text { for pilot }\end{array}$ & Yes \\
\hline $\begin{array}{l}\text { 3. Ng } \\
(2019)\end{array}$ & $\begin{array}{l}\text { Yes }(12 \mathrm{~m}) \\
\text { CGM- FoH/ HbA1c }\end{array}$ & $\begin{array}{l}\text { Yes: }<18 \text { years } \\
\text { Minimum } 12 \mathrm{~m} \text { CGM } \\
\text { English speaking }\end{array}$ & $\begin{array}{l}\text { Yes- of UK clinic population } \\
\text { Limited demographics }\end{array}$ & Unclear & $\begin{array}{l}\text { No }(n=16) \\
\text { No power calculation }\end{array}$ & $\begin{array}{l}\text { Clear intervention } \\
58 \% \text { uncompliant }\end{array}$ \\
\hline $\begin{array}{l}\text { 4.Kamps } \\
(2010)\end{array}$ & $\begin{array}{l}\text { Yes } \\
\text { Trauma- FoH/Anxiety } \\
\text { HbA1c }\end{array}$ & $\begin{array}{l}\text { Yes: 8-16 years } \\
\text { Minimum 6m T1DM } \\
\text { No chronic illness }\end{array}$ & $\begin{array}{l}\text { Yes- of US clinic population } \\
\text { Higher income, education, } \\
\text { duration in } x 2 \text { completers }\end{array}$ & $\begin{array}{l}\text { Most } \\
89 \% \text { recruitment }\end{array}$ & $\begin{array}{l}\text { Moderate }(n=158) \\
\text { No power calculation }\end{array}$ & $\begin{array}{l}\text { No } \\
\text { No measure of } \\
\text { exposure/ stress }\end{array}$ \\
\hline $\begin{array}{l}\text { 5.Muller- } \\
\text { Godeffroy } \\
\text { (2009) }\end{array}$ & $\begin{array}{l}\text { Yes }(6 \mathrm{~m}) \\
\text { CSII-Psychosocial }\end{array}$ & $\begin{array}{l}\text { Yes: } 4-16 \text { years } \\
\text { Minimum } 6 \mathrm{~m} \text { T1DM } \\
\text { Sufficient literacy }\end{array}$ & $\begin{array}{l}\text { Yes-18 centres in Germany } \\
\text { Limited demographics }\end{array}$ & Unclear & $\begin{array}{l}\text { Almost }(n=117) \\
80 \% \text { power }=120\end{array}$ & Yes \\
\hline
\end{tabular}

\begin{tabular}{|c|c|c|c|c|c|c|}
\hline Study & 7 & 8 & 9 & 10 & 11 & 12 \\
\hline $\begin{array}{l}\text { First Author } \\
\text { Publication Year }\end{array}$ & $\begin{array}{l}\text { Outcomes defined, } \\
\text { valid, reliable \& } \\
\text { consistent }\end{array}$ & $\begin{array}{l}\text { Assessors } \\
\text { blinded }\end{array}$ & $\begin{array}{l}\text { Loss to follow up }<20 \% \\
\text { and accounted for }\end{array}$ & $\begin{array}{l}\mathrm{P} \text { values provided for } \\
\text { pre- to post- changes }\end{array}$ & $\begin{array}{l}\text { Outcomes } \\
\text { measured } \\
\text { multiple times }\end{array}$ & $\begin{array}{l}\text { Group-level } \\
\text { intervention v. } \\
\text { individual data }\end{array}$ \\
\hline $\begin{array}{l}\text { 1.Al Hayek } \\
\text { (2017) }\end{array}$ & $\begin{array}{l}\text { Yes } \\
\text { HFS-C, Peds QL }\end{array}$ & No & $\begin{array}{l}\text { Yes } \\
\text { No loss to follow up cited }\end{array}$ & Yes & No & NA \\
\hline $\begin{array}{l}\text { 2.Cai } \\
\text { (2017) }\end{array}$ & $\begin{array}{l}\text { Yes } \\
\text { HFS, Peds QL }\end{array}$ & No & $\begin{array}{l}\text { Accounted for but high: } \\
34 \% \text { loss to follow up }\end{array}$ & $\begin{array}{l}\text { No } \\
\text { Pilot intervention }\end{array}$ & $\begin{array}{l}\text { Yes- outcomes at } \\
1 \text { and } 3 m\end{array}$ & NA \\
\hline $\begin{array}{l}\text { 3. } \mathrm{Ng} \\
(2019)\end{array}$ & $\begin{array}{l}\text { Yes } \\
\text { HFS/ HbA1c }\end{array}$ & No & $\begin{array}{l}\text { Yes } \\
\text { Only } 8 \% \text { loss to follow up }\end{array}$ & Yes & $\begin{array}{l}\text { Yes- HbA1c at } \\
3,6,9,12 \mathrm{~m}\end{array}$ & NA \\
\hline $\begin{array}{l}\text { 4.Kamps } \\
\text { (2010) }\end{array}$ & $\begin{array}{l}\text { Yes } \\
\text { CHI, RCMAS/ HbA1c }\end{array}$ & No & $\begin{array}{l}\text { Accounted for but high: } \\
25 \% \text { loss to follow up }\end{array}$ & Yes & No & NA \\
\hline $\begin{array}{l}\text { 5.Muller- } \\
\text { Godeffroy } \\
\text { (2009) }\end{array}$ & $\begin{array}{l}\text { Yes } \\
\text { HRQOL, PIP, HFS } \\
\text { HbA1c }\end{array}$ & No & $\begin{array}{l}\text { Accounted for but high: } \\
23 \% \text { in CYP } \\
18 \% \text { in parents }\end{array}$ & Yes & No & NA \\
\hline
\end{tabular}


Appendix 2. Quality Assessment Tables (continued)

D. Literature Reviews/Systematic Reviews ( $n=2)$

CASP Systematic Review Checklist (CASP, 2018)

1. Did the review address a clearly focused question?

2. Did the authors look for the right type of papers?

3. Do you think all the important, relevant studies were included?

4. Did the review's authors do enough to assess quality of the included studies?

5. If the results of the review have been combined, was it reasonable to do so?

6. What are the overall results of the review?

7. How precise are the results?

8. Can the results be applied to the local population?

9. Were all important outcomes considered?

10. Are the benefits worth the harms and costs?

\begin{tabular}{|l|l|l|l|l|l|}
\hline Study & $\mathbf{1}$ & $\mathbf{2}$ & $\mathbf{3}$ & $\mathbf{4}$ & $\mathbf{5}$ \\
\hline $\begin{array}{l}\text { First Author } \\
\text { Publication Year }\end{array}$ & $\begin{array}{l}\text { Focused } \\
\text { question }\end{array}$ & $\begin{array}{l}\text { Appropriate } \\
\text { papers }\end{array}$ & Important relevant studies included & Quality assessment & Results combination \\
\hline $\begin{array}{l}\text { 1.Barnard } \\
(2010)\end{array}$ & $\begin{array}{l}\text { Yes } \\
\text { FoH in parents of } \\
\text { young children }\end{array}$ & $\begin{array}{l}\text { Yes } \\
\text { Cross-sectional }\end{array}$ & $\begin{array}{l}\text { Mostly: CRD principles; 2 reviewers } \\
\text { Multiple databases, meeting abstracts, } \\
\text { bibliographies, experts, } \\
\text { But only 6 studies; nil interventional }\end{array}$ & $\begin{array}{l}\text { Yes: Crombie criteria } \\
\text { 2 reviewers } \\
\text { X1 7/7 quality indicators } \\
\text { x3 met 6/7; x2 met 4/7 }\end{array}$ & $\begin{array}{l}\text { No } \\
\text { Dissimilar cohorts/ outcomes } \\
\text { Lack of data } \\
\text { Narrative synthesis }\end{array}$ \\
\hline $\begin{array}{l}\text { 2.Driscoll } \\
(2016)\end{array}$ & $\begin{array}{l}\text { Yes } \\
\text { FoH in CYP and } \\
\text { parents } \\
\text { Literature review }\end{array}$ & $\begin{array}{l}\text { Yes } \\
\text { Cross-sectional }\end{array}$ & Greater breadth- 16 studies & Unclear & Norrative analysis \\
\hline
\end{tabular}

\begin{tabular}{|l|l|l|l|l|l|}
\hline Study & $\mathbf{6}$ & $\mathbf{7}$ & $\mathbf{8}$ & $\mathbf{9}$ & $\mathbf{1 0}$ \\
\hline $\begin{array}{l}\text { First Author } \\
\text { Publication Year }\end{array}$ & Overall results & Result precision & Locally applicable & $\begin{array}{l}\text { All-important outcomes } \\
\text { considered }\end{array}$ & $\begin{array}{l}\text { Benefits worth } \\
\text { harms and costs }\end{array}$ \\
\hline $\begin{array}{l}\text { 1.Barnard } \\
(2010)\end{array}$ & $\begin{array}{l}\text { Parental FoH/ anxiety/ } \\
\text { depression are common } \\
\text { Hypoglycaemia severity } \\
\text { predicts FoH }>\text { frequency }\end{array}$ & $\begin{array}{l}\text { Some results precise } \\
\text { p values given }\end{array}$ & $\begin{array}{l}\text { Somewhat } \\
\text { 4 studies representative } \\
\text { Mostly US studies; similar to UK }\end{array}$ & $\begin{array}{l}\text { Yes } \\
\text { Except intervention/ } \\
\text { education }\end{array}$ & NA \\
\hline $\begin{array}{l}\text { 2.Driscoll } \\
(2016)\end{array}$ & $\begin{array}{l}\text { Parent report of SH was the } \\
\text { most common predictor o } \\
\text { Most studies failed to find } \\
\text { an association with HbA1c }\end{array}$ & Unclear & $\begin{array}{l}\text { Somewhat } \\
16 \text { studies- mainly US/ European }\end{array}$ & $\begin{array}{l}\text { Yes Associated factors, } \\
\text { behavioural interventions, } \\
\text { technology }\end{array}$ & NA \\
\hline
\end{tabular}


Appendix 3. Data Extraction Tables

\begin{tabular}{|c|c|c|c|c|c|c|c|c|c|c|}
\hline 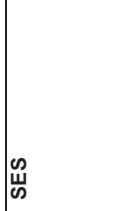 & 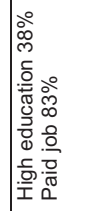 & $\frac{\alpha}{z}$ & 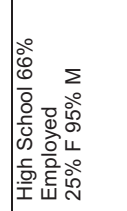 & 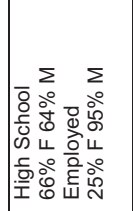 & 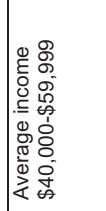 & 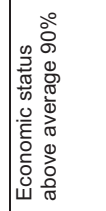 & $\frac{\alpha}{2}$ & $\frac{\alpha}{2}$ & 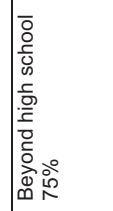 & 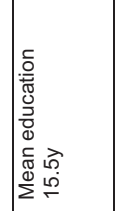 \\
\hline 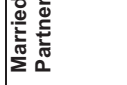 & ळ̊ & $\frac{\alpha}{z}$ & $\frac{\alpha}{z}$ & $\frac{\alpha}{z}$ & $\frac{\alpha}{z}$ & ஓ̊ & $\frac{\alpha}{2}$ & $\frac{\alpha}{2}$ & 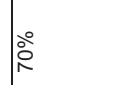 & $\frac{\alpha}{2}$ \\
\hline 量 & 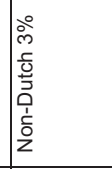 & 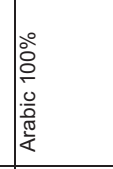 & 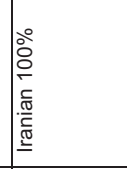 & 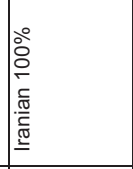 & 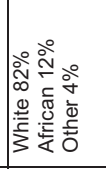 & $\frac{\alpha}{Z}$ & 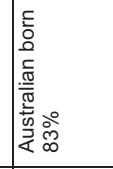 & 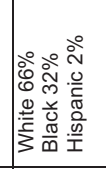 & 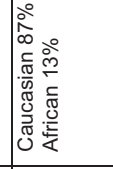 & 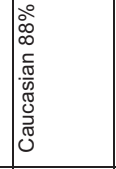 \\
\hline 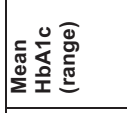 & 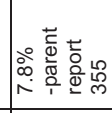 & $\frac{x}{z}$ & 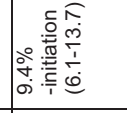 & 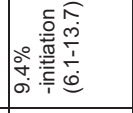 & 总 & 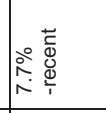 & 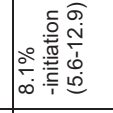 & 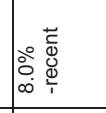 & 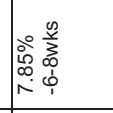 & $\begin{array}{l}\stackrel{0}{0} \\
\vdots \\
\infty \\
\infty \\
0\end{array}$ \\
\hline 宫 & 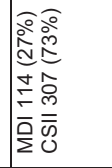 & 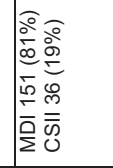 & 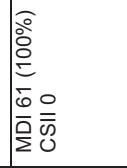 & 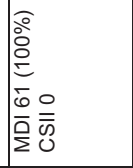 & $\frac{\alpha}{z}$ & 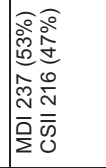 & 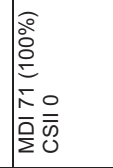 & 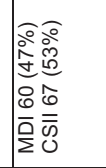 & 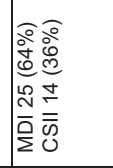 & 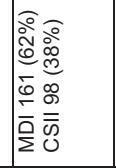 \\
\hline 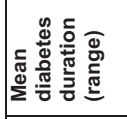 & 它 & 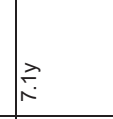 & 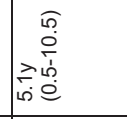 & 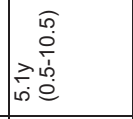 & $\begin{array}{l}\overrightarrow{\widehat{y}} \\
\substack{0 \\
0} \\
\end{array}$ & बे & 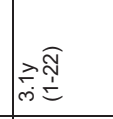 & ج্তে & 旁 & 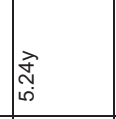 \\
\hline 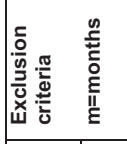 & $\frac{x}{2}$ & 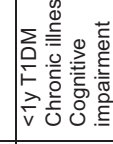 & 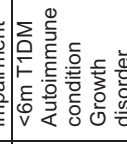 & $\begin{array}{l}0 \\
0\end{array}$ & 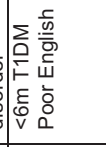 & $\frac{\alpha}{z}$ & $\frac{\alpha}{z}$ & 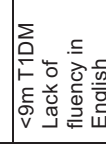 & 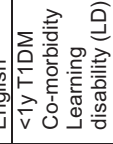 & \\
\hline 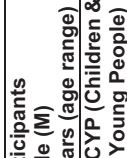 & 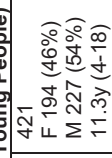 & 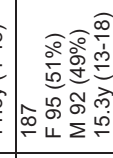 & 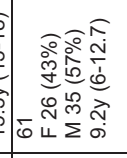 & 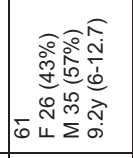 & 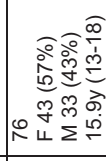 & 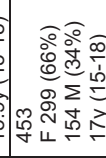 & 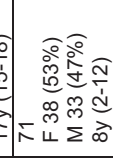 & 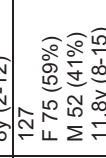 & 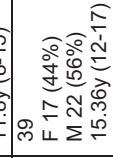 & 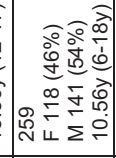 \\
\hline 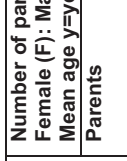 & 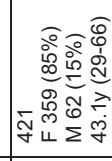 & $\frac{z}{z}$ & 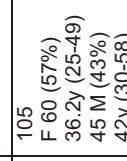 & 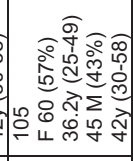 & & $\mathbb{z}$ & 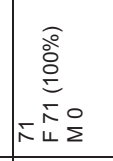 & $\frac{\alpha}{z}$ & 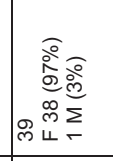 & 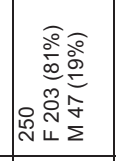 \\
\hline 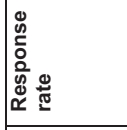 & 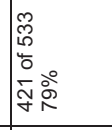 & $\frac{\alpha}{z}$ & 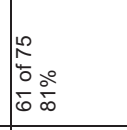 & 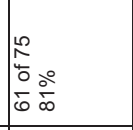 & 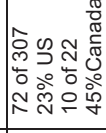 & 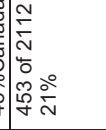 & 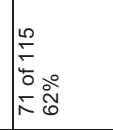 & $\frac{\alpha}{z}$ & 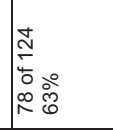 & $\frac{\alpha}{2}$ \\
\hline 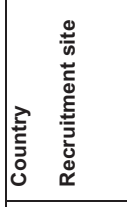 & 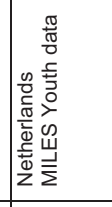 & 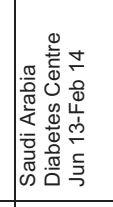 & 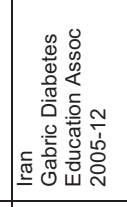 & 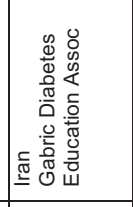 & 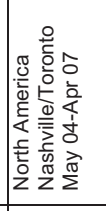 & 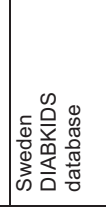 & 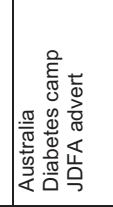 & 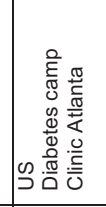 & 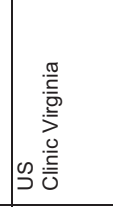 & 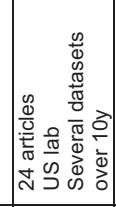 \\
\hline 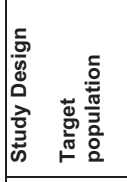 & 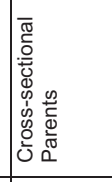 & 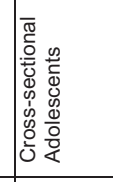 & 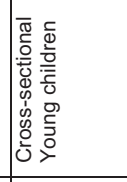 & 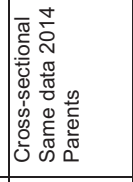 & 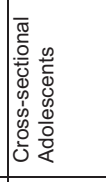 & 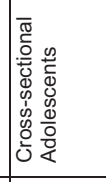 & 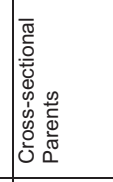 & 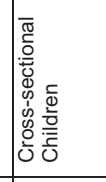 & 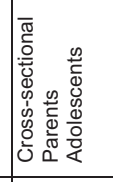 & 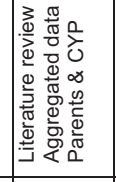 \\
\hline 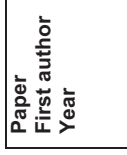 & 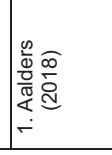 & 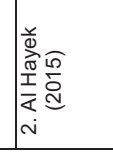 & 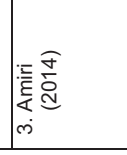 & 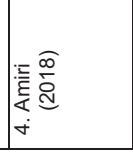 & 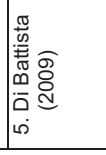 & 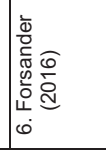 & 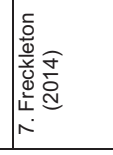 & 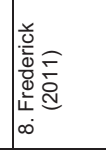 & 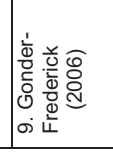 & 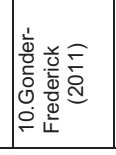 \\
\hline
\end{tabular}


Appendix 3. Data Extraction Tables (continued)

\begin{tabular}{|c|c|c|c|c|c|c|c|c|c|c|c|c|}
\hline 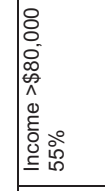 & $\frac{\alpha}{2}$ & 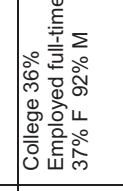 & $\frac{\alpha}{z}$ & 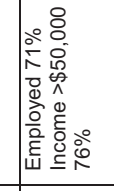 & $\frac{x}{z}$ & 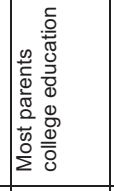 & $\frac{\alpha}{z}$ & 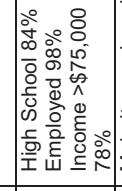 & & 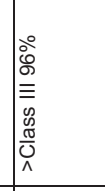 & & 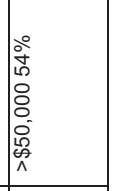 \\
\hline$\frac{p}{z}$ & $\frac{\alpha}{2}$ & 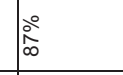 & $\frac{\alpha}{z}$ & 这 & $\frac{\alpha}{z}$ & $\frac{\alpha}{z}$ & $\frac{\alpha}{z}$ & 吕 & & ڤે̊ & 号 & ఏ̊ \\
\hline 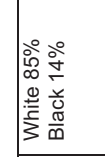 & $\frac{d}{20}$ & $\frac{\alpha}{z}$ & $\frac{\alpha}{z}$ & 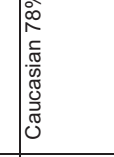 & $\frac{\alpha}{z}$ & 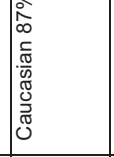 & $\frac{\alpha}{z}$ & 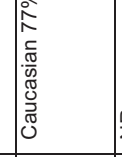 & $\frac{x}{z}$ & 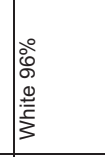 & & 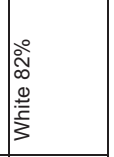 \\
\hline 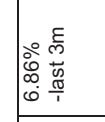 & 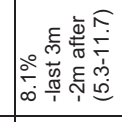 & 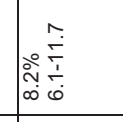 & 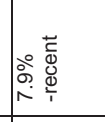 & 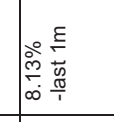 & 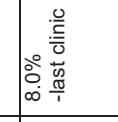 & $\frac{\alpha}{2}$ & : & 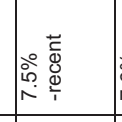 & 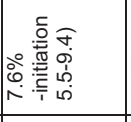 & 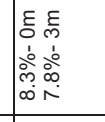 & 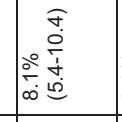 & 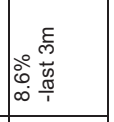 \\
\hline 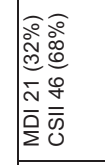 & 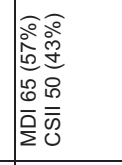 & 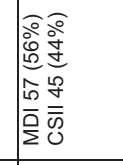 & 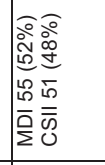 & 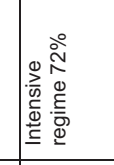 & 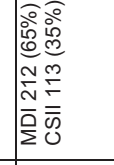 & $\frac{\alpha}{2}$ & 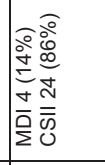 & 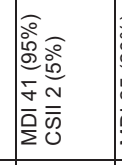 & 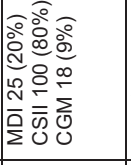 & 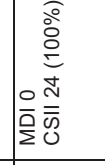 & 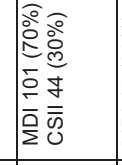 & 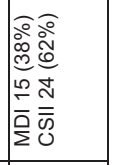 \\
\hline 字 & 趂 & 勇矢 & 勇 & 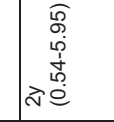 & 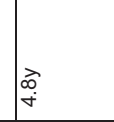 & $\frac{\alpha}{2}$ & ִָָ & ?ָे & $\frac{\frac{Y}{E}}{\Sigma}$ & $\stackrel{P}{c}$ & 熟 & $\frac{\alpha}{2}$ \\
\hline 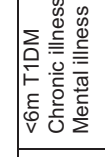 & $\frac{\infty}{2}$ & $\frac{\alpha}{z}$ & 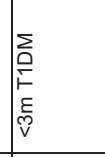 & & & $\frac{\alpha}{z}$ & $\frac{\alpha}{z}$ & 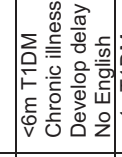 & & 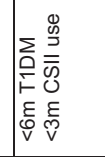 & & 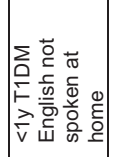 \\
\hline 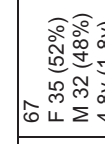 & 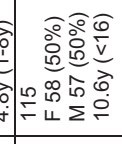 & 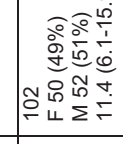 & 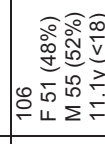 & & & 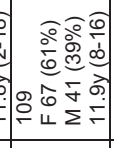 & 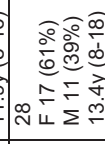 & & 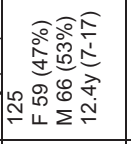 & 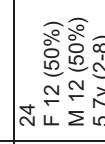 & 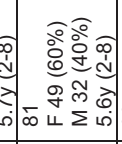 & 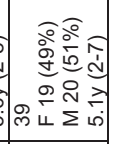 \\
\hline 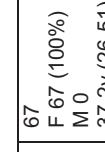 & 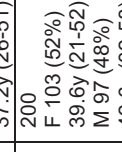 & 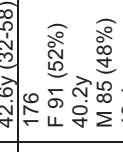 & 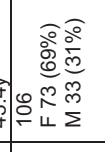 & 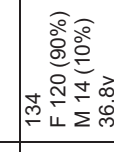 & & $\frac{s}{z}$ & 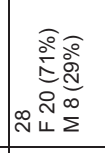 & 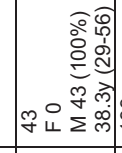 & 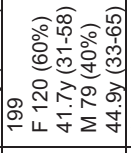 & 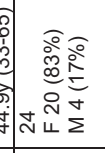 & 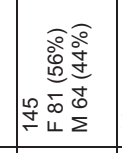 & 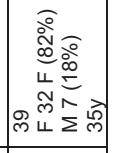 \\
\hline & | & 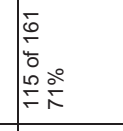 & $\frac{\underline{\alpha}}{z}$ & 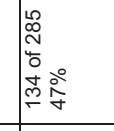 & 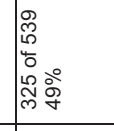 & 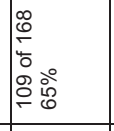 & 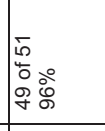 & 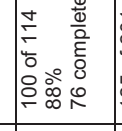 & 离。 & 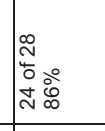 & 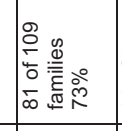 & \\
\hline 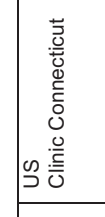 & 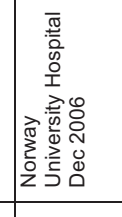 & 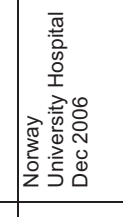 & 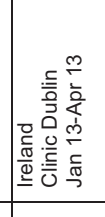 & 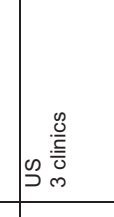 & 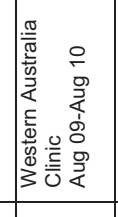 & 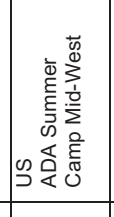 & 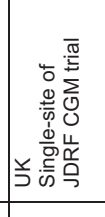 & 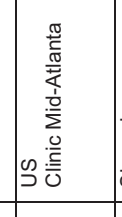 & 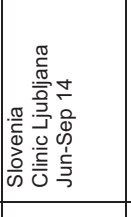 & 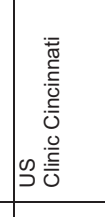 & 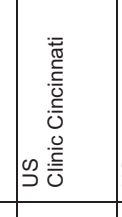 & 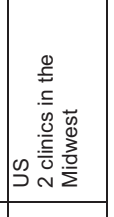 \\
\hline 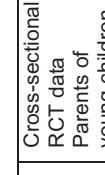 & $\begin{array}{l}0.0 \\
0\end{array}$ & 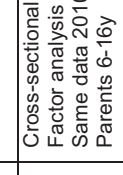 & 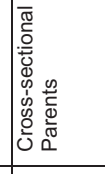 & 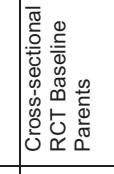 & 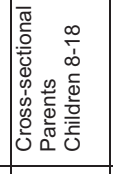 & 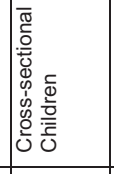 & 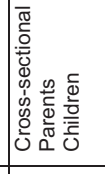 & 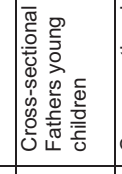 & 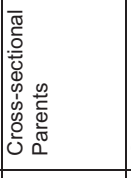 & 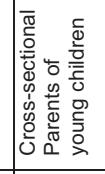 & 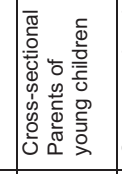 & 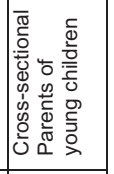 \\
\hline & 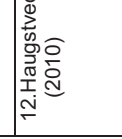 & 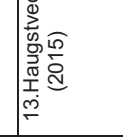 & 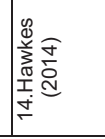 & $\mid$ & 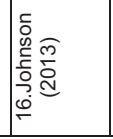 & 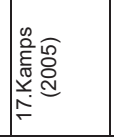 & 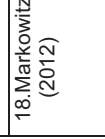 & 离 & & 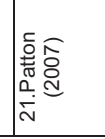 & $\mid$ & 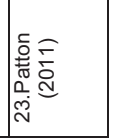 \\
\hline
\end{tabular}


Appendix 3. Data Extraction Tables (continued)

\begin{tabular}{|c|c|c|c|c|c|c|c|c|c|c|c|c|c|}
\hline$\frac{x}{2}$ & 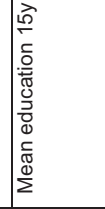 & 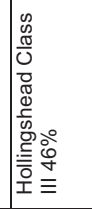 & 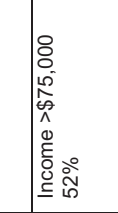 & $\frac{\alpha}{z}$ & $\frac{\alpha}{z}$ & $\frac{\alpha}{z}$ & $\frac{\alpha}{2}$ & 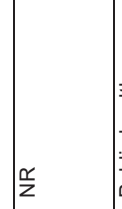 & & 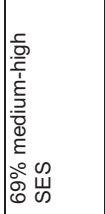 & 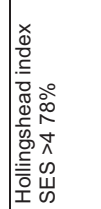 & $\frac{\alpha}{z}$ & $\frac{\alpha}{2}$ \\
\hline$\frac{\mathrm{u}}{\mathrm{z}}$ & 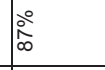 & 獘 & $\frac{\alpha}{2}$ & 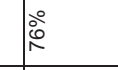 & $\frac{\alpha}{z}$ & $\frac{\alpha}{2}$ & $\frac{\alpha}{2}$ & $\frac{\alpha}{2}$ & 竞 & $\frac{\alpha}{z}$ & $\stackrel{\circ}{\frac{\circ}{\infty}}$ & $\frac{\alpha}{z}$ & $\frac{\alpha}{2}$ \\
\hline $\bar{\xi}$ & 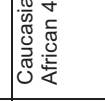 & 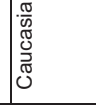 & 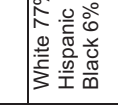 & $\frac{\alpha}{2}$ & $\frac{\alpha}{2}$ & $\frac{\alpha}{2}$ & $\frac{\alpha}{2}$ & $\frac{\alpha}{2}$ & 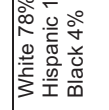 & $\frac{\alpha}{z}$ & 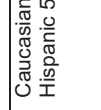 & $\frac{\alpha}{z}$ & $\frac{\alpha}{z}$ \\
\hline 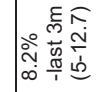 & 商 & 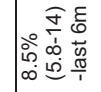 & 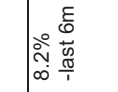 & 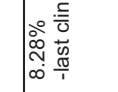 & 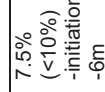 & & & 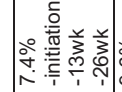 & 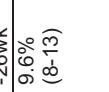 & 号 & 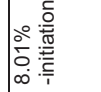 & 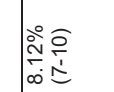 & 总 \\
\hline 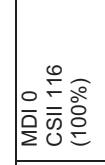 & 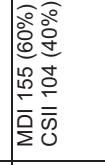 & 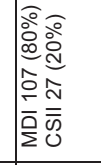 & 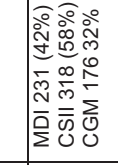 & $\frac{\alpha}{2}$ & 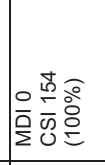 & 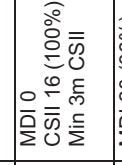 & 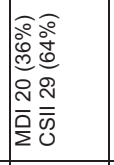 & 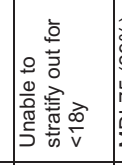 & 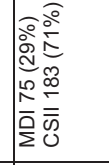 & 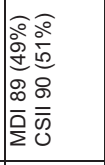 & 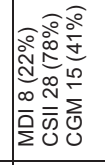 & 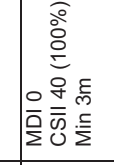 & 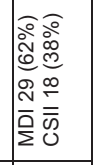 \\
\hline & 京 & 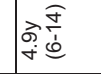 & ج্রi & 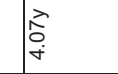 & 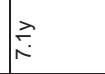 & זָר & శె & & 勇 & 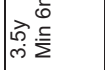 & $\frac{\alpha}{2}$ & के & $\frac{\alpha}{2}$ \\
\hline 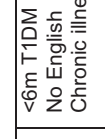 & 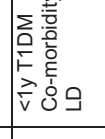 & 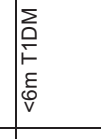 & 旁 & 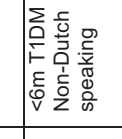 & 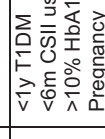 & & & 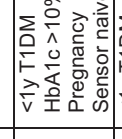 & & & & & 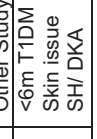 \\
\hline & & & & & & & & & & & & & 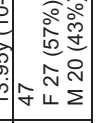 \\
\hline 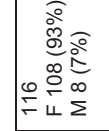 & 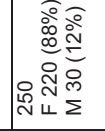 & 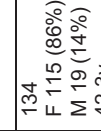 & & 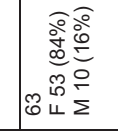 & $\frac{s}{z}$ & 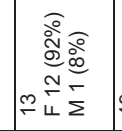 & g & $\approx \widetilde{\approx} \frac{\alpha}{z}$ & 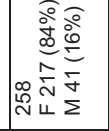 & $\frac{\alpha}{2}$ & 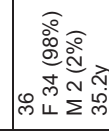 & & $\frac{s}{z}$ \\
\hline$z$ & $\frac{s}{2}$ & 离 & 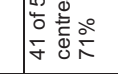 & 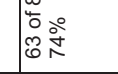 & $\frac{\alpha}{2}$ & & $\frac{\alpha}{2}$ & $\frac{\pi}{40}$ & & 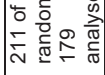 & & & $\frac{\alpha}{2}$ \\
\hline 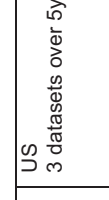 & 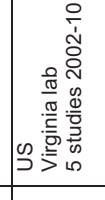 & 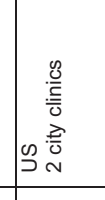 & 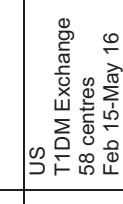 & 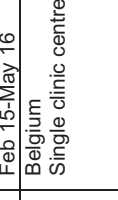 & 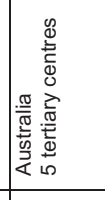 & 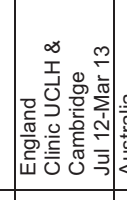 & & 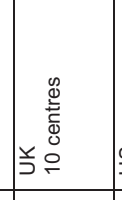 & 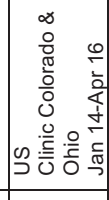 & 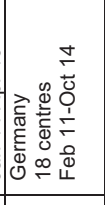 & 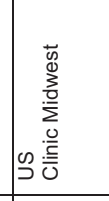 & 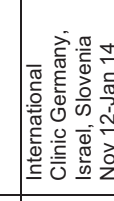 & 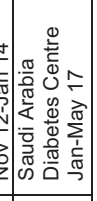 \\
\hline 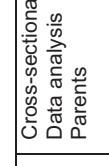 & 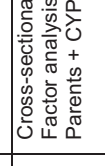 & 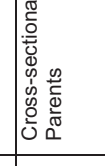 & 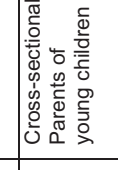 & 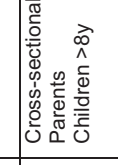 & 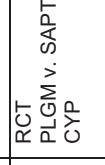 & & 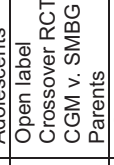 & 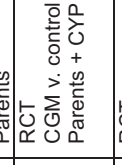 & 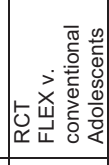 & 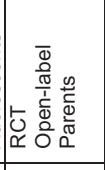 & 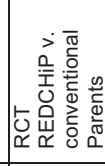 & 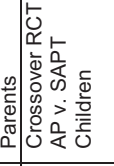 & 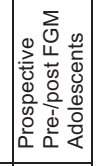 \\
\hline 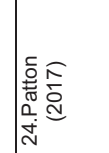 & $\mid$ & 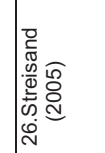 & 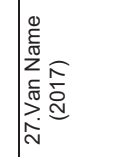 & $\mid$ & 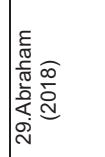 & 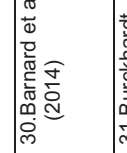 & 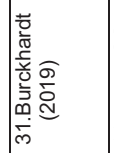 & $\mid$ & 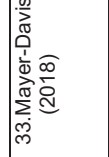 & 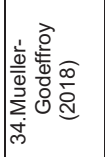 & 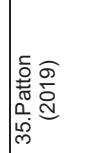 & 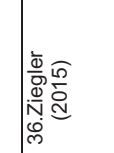 & 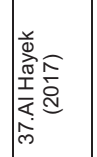 \\
\hline
\end{tabular}


Appendix 3. Data Extraction Tables (continued)

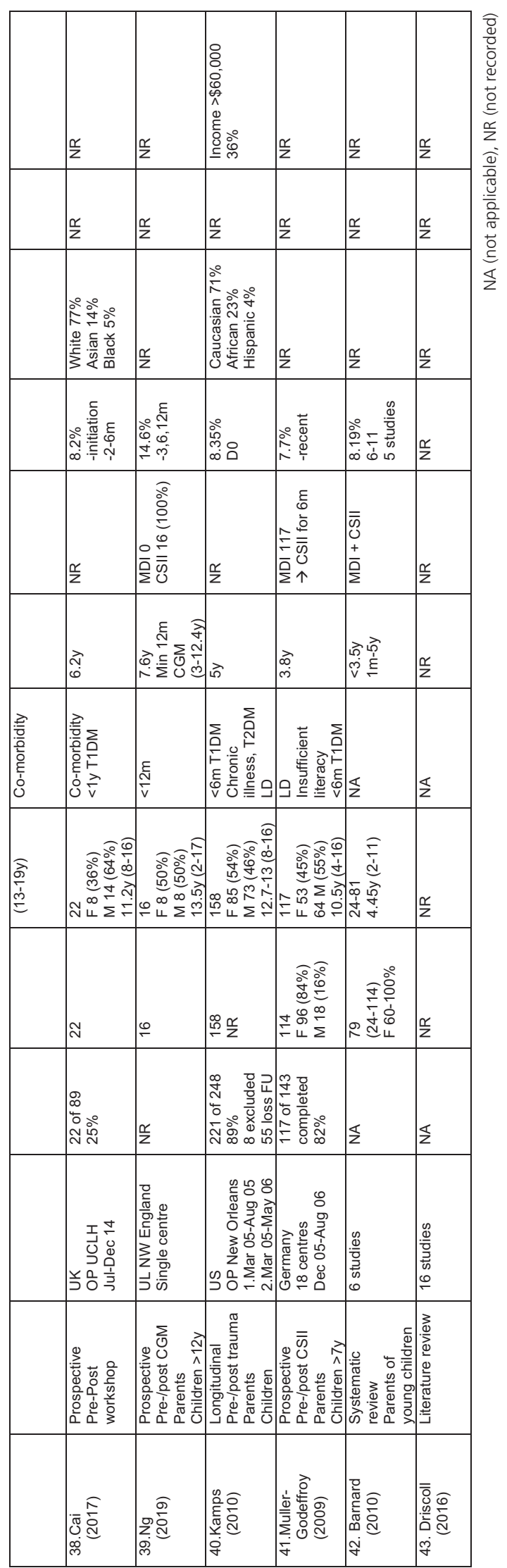


Appendix 3. Data Extraction Tables (continued)

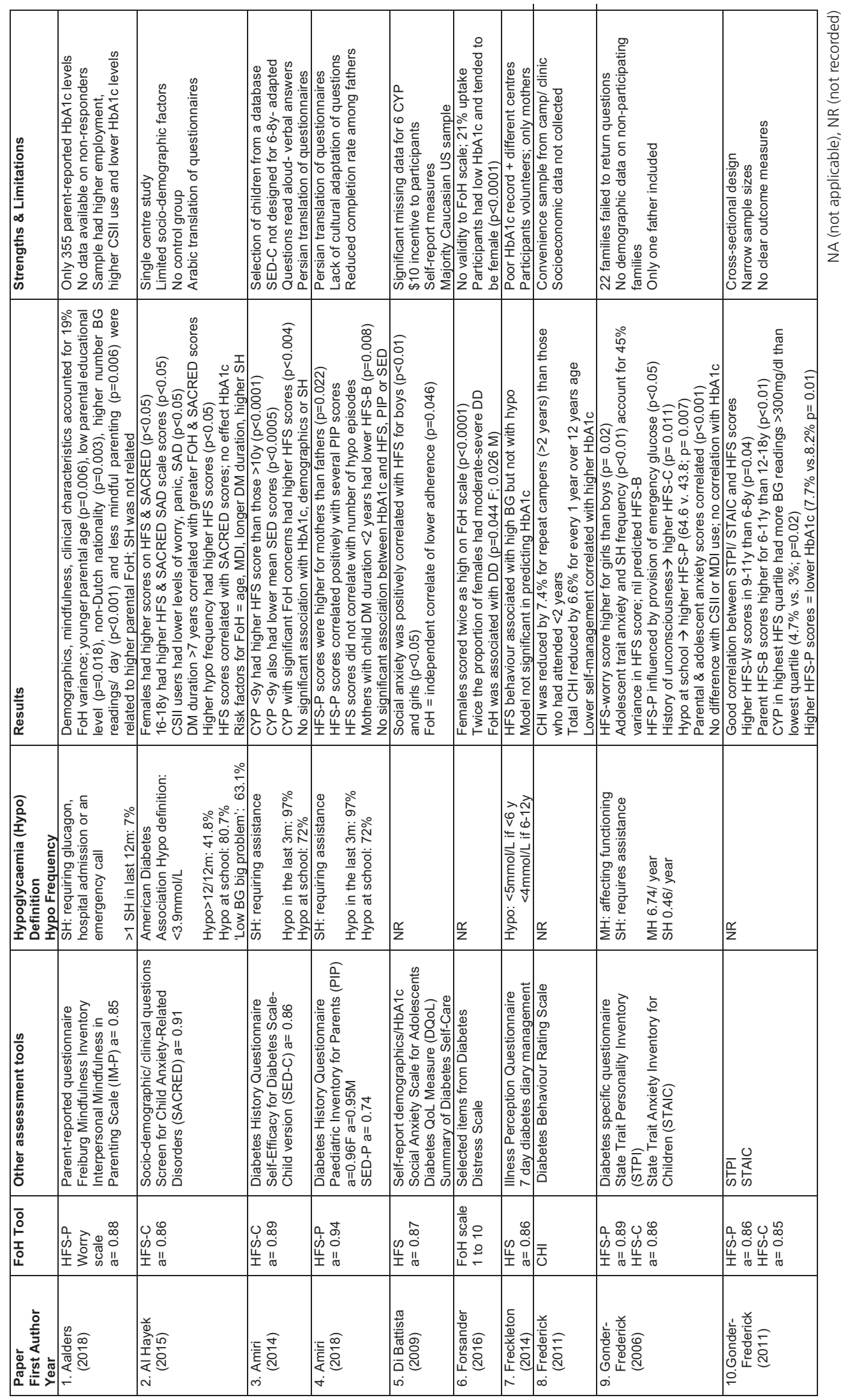


Appendix 3. Data Extraction Tables (continued)

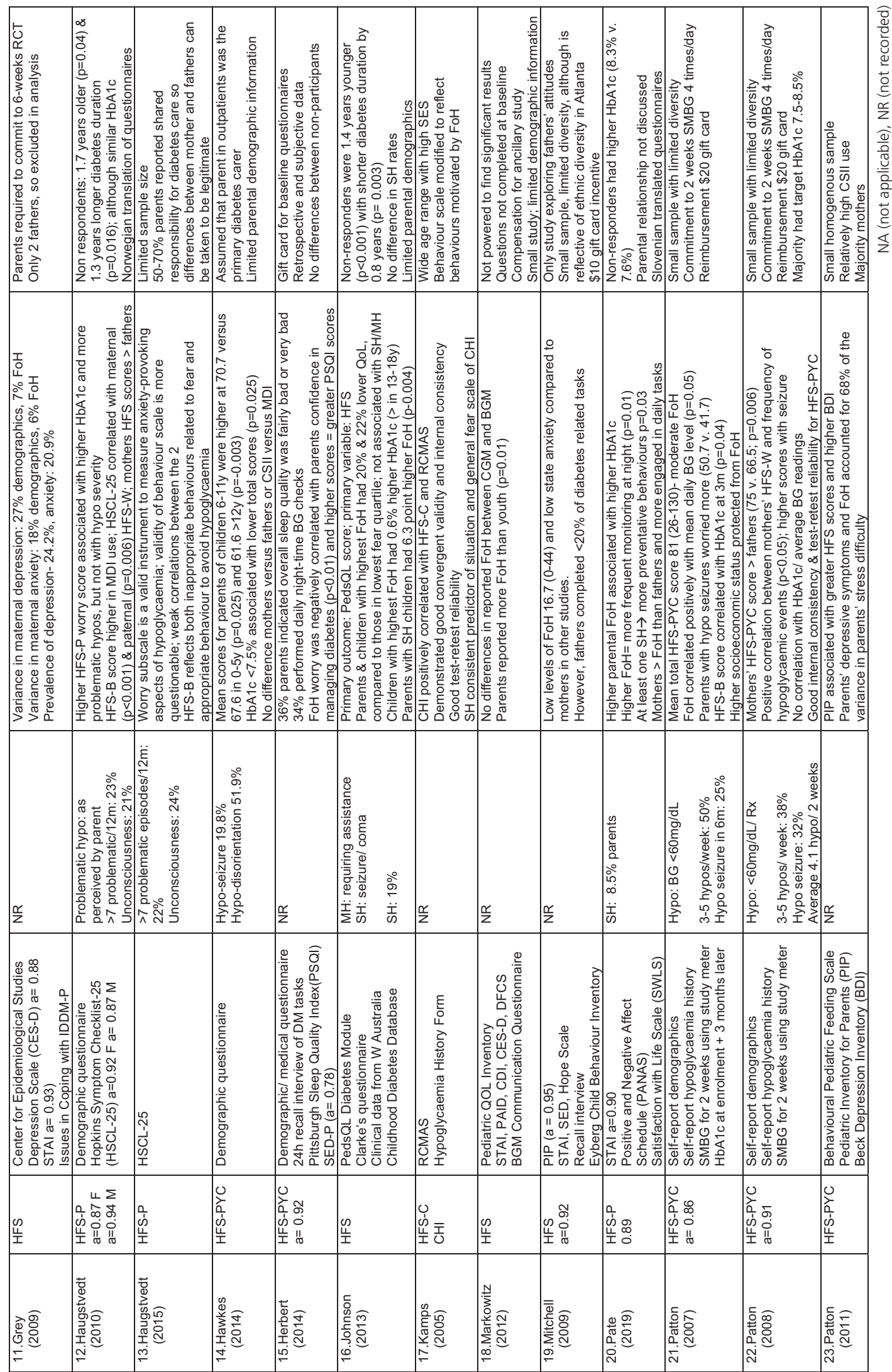


Appendix 3. Data Extraction Tables (continued)
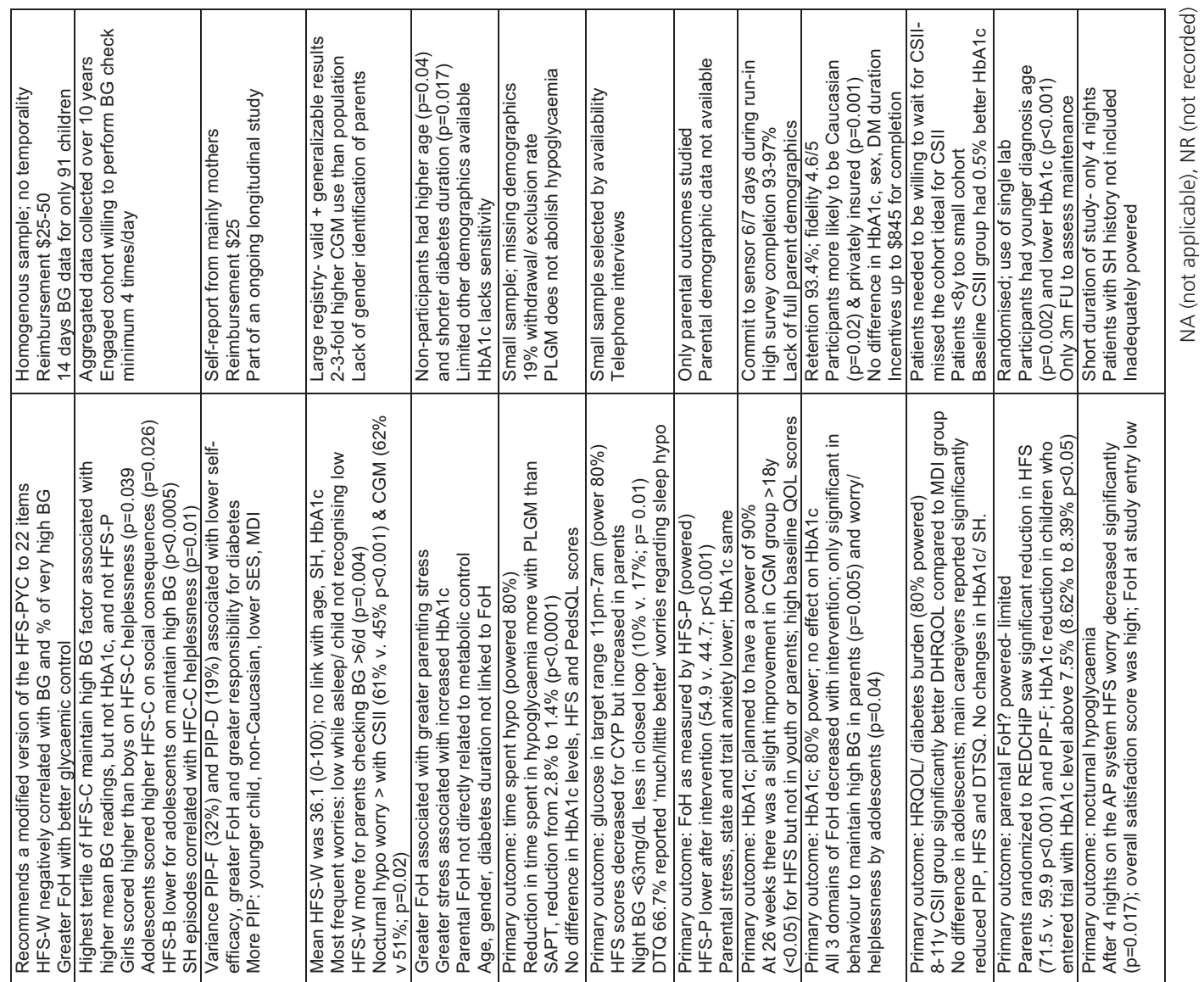

\begin{tabular}{|c|c|c|c|c|c|c|c|c|c|c|c|c|}
\hline$\frac{\alpha}{z}$ & 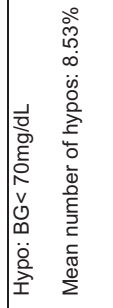 & $\frac{\alpha}{z}$ & 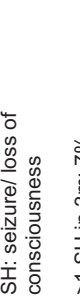 & 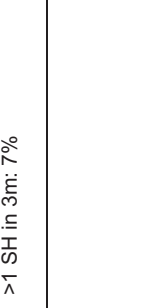 & 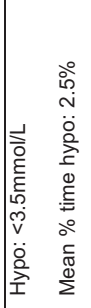 & 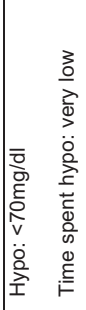 & $\frac{\alpha}{z}$ & 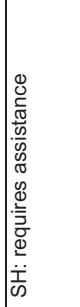 & 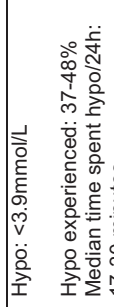 & & 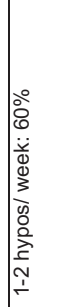 & $\frac{\alpha}{z}$ \\
\hline 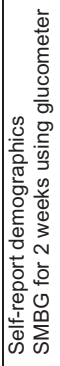 & 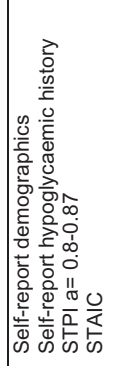 & 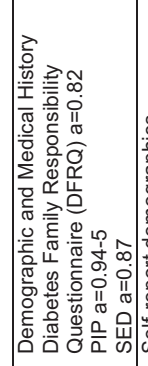 & 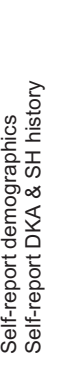 & 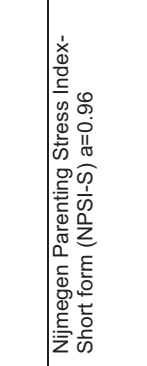 & 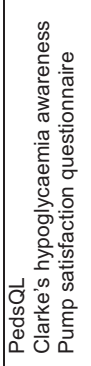 & 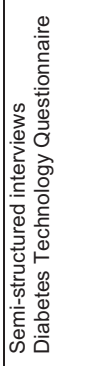 & 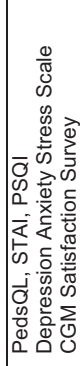 & 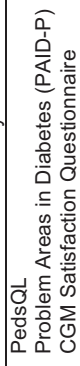 & 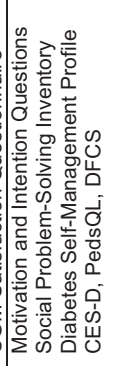 & 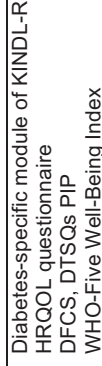 & $\frac{\alpha}{0}$ & 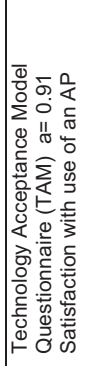 \\
\hline 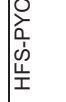 & 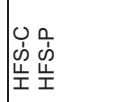 & 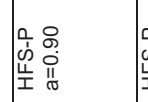 & 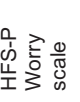 & 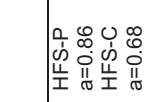 & & $\mid \begin{array}{l}\infty \\
\\
\\
\end{array}$ & $\mid \begin{array}{l}0 \\
\dot{\omega} \\
\frac{u}{I}\end{array}$ & 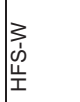 & $\begin{array}{l}00 \\
\frac{u}{I}\end{array}$ & $\begin{array}{l}0 \\
0 \dot{j} \\
\frac{1}{I} \\
\end{array}$ & 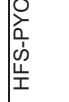 & 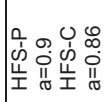 \\
\hline 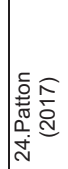 & 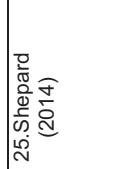 & 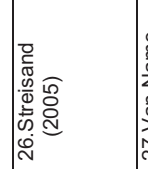 & 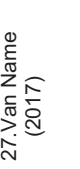 & 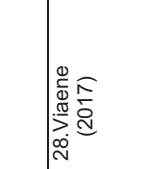 & 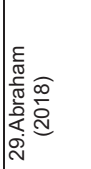 & 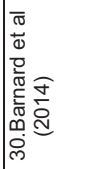 & 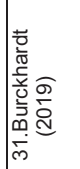 & 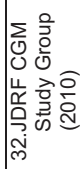 & 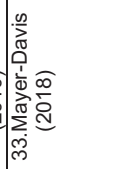 & 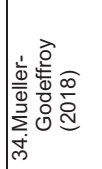 & 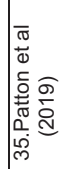 & 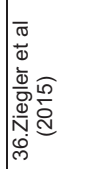 \\
\hline
\end{tabular}


Appendix 3. Data Extraction Tables (continued)

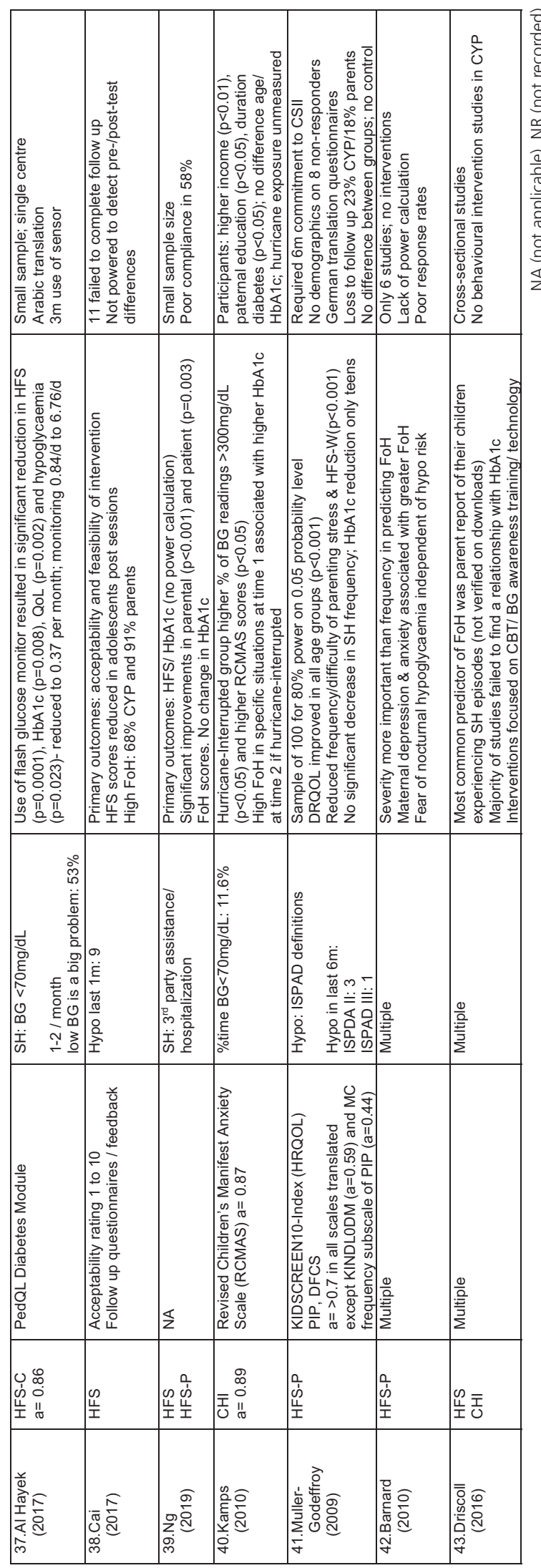

